\title{
TORIC DEGENERATIONS OF INTEGRABLE SYSTEMS ON GRASSMANNIANS AND POLYGON SPACES
}

\author{
YUICHI NOHARA AND KAZUSHI UEDA
}

\begin{abstract}
We introduce a completely integrable system on the Grassmannian of 2-planes in an $n$-space associated with any triangulation of a polygon with $n$ sides, and we compute the potential function for its Lagrangian torus fiber. The moment polytopes of this system for different triangulations are related by an integral piecewise-linear transformation, and the corresponding potential functions are related by its geometric lift in the sense of Berenstein and Zelevinsky.
\end{abstract}

\section{$\S 1$. Introduction}

Let $n$ be an integer greater than 2 , and let $\boldsymbol{r}=\left(r_{1}, \ldots, r_{n}\right)$ be a sequence of positive real numbers satisfying

$$
r_{i}<r_{1}+\cdots+r_{i-1}+r_{i+1}+\cdots+r_{n}
$$

for each $i=1, \ldots, n$. The polygon space $\mathcal{M}_{\boldsymbol{r}}$ is defined by

$$
\mathcal{M}_{r}=\left\{\boldsymbol{x}=\left(x_{1}, \ldots, x_{n}\right) \in \prod_{i=1}^{n} S^{2}\left(r_{i}\right) \mid x_{1}+\cdots+x_{n}=0\right\} / \mathrm{SO}(3),
$$

where $S^{2}\left(r_{i}\right) \subset \mathbb{R}^{3}$ is the 2 -sphere of radius $r_{i}$ centered at the origin and where $\mathrm{SO}(3)$ acts diagonally on $\prod_{i=1}^{n} S^{2}\left(r_{i}\right)$. A point $\boldsymbol{x} \in \mathcal{M}_{\boldsymbol{r}}$ is regarded as a congruence class of a closed spatial polygon with sides $x_{1}, \ldots, x_{n}$. Condition (1.1) implies that $\mathcal{M}_{r} \neq \emptyset$ and that $\operatorname{dim}_{\mathbb{R}} \mathcal{M}_{r}=2(n-3)$. The variety $\mathcal{M}_{\boldsymbol{r}}$ is singular if and only if $\sum_{i=1}^{n} \epsilon_{i} r_{i}=0$ for some $\left(\epsilon_{1}, \ldots, \epsilon_{n}\right) \in\{ \pm 1\}^{n}$, and the singular locus of $\mathcal{M}_{\boldsymbol{r}}$ consists of $\boldsymbol{x}=\left(x_{1}, \ldots, x_{n}\right) \in \mathcal{M}_{\boldsymbol{r}}$ satisfying

Received July 5, 2012. Revised February 18, 2013. Accepted May 14, 2013.

First published online March 3, 2014.

2010 Mathematics Subject Classification. Primary 37J35; Secondary 53D37.

Both authors' work was partially supported by Grants-in-Aid for Young Scientists 19740025 and 23740055 (Nohara) and 20740037 (Ueda) from the Japan Society for the Promotion of Science. 
$\sum_{i=1}^{n} \epsilon_{i} x_{i}=0$. In particular, the polygon space $\mathcal{M}_{r}$ has at worst isolated singularities for any $\boldsymbol{r}$. The polygon space $\mathcal{M}_{r}$ has a natural structure of a projective (and hence Kähler) variety coming from the identification

$$
\mathcal{M}_{r} \cong\left(\mathbb{C P}^{1}\right)^{n} / / \operatorname{PGL}(2, \mathbb{C}) .
$$

The geometric invariant theory (GIT) quotient on the right-hand side is a compactification of the configuration space of $n$ points on $\mathbb{C P}$, which has a long history of investigation going back to the nineteenth century. Furthermore, the Gelfand-MacPherson correspondence (see [GM]) gives an isomorphism between the polygon space and the symplectic reduction of the Grassmannian $\operatorname{Gr}(2, n)$ of 2-planes in $\mathbb{C}^{n}$ by a maximal torus $T_{U(n)}$ of $U(n)$ :

$$
\mathcal{M}_{r} \cong \operatorname{Gr}(2, n) / / T_{U(n)} .
$$

It is also known (see $[\mathrm{Fo}],[\mathrm{HMM}],[\mathrm{J}],[\mathrm{MP}],[\mathrm{T}]$ ) that $\mathcal{M}_{\boldsymbol{r}}$ is symplectomorphic to the moduli space of parabolic $\mathrm{SU}(2)$-bundles on $\mathbb{C P}^{1}$ for sufficiently small $\boldsymbol{r}$.

Recall that a completely integrable system on a symplectic manifold $(X, \omega)$ of dimension $2 N$ is an $N$-tuple of functions

$$
\Phi=\left(\varphi_{1}, \ldots, \varphi_{N}\right): X \longrightarrow \mathbb{R}^{N},
$$

which are functionally independent (i.e., $d \varphi_{1}, \ldots, d \varphi_{N}$ are linearly independent) on an open dense subset and mutually Poisson commutative; $\left\{\varphi_{i}, \varphi_{j}\right\}=0$ for any $i, j$. The Arnold-Liouville theorem states that generic fibers of $\Phi$ are Lagrangian tori if the fibers are compact and connected. The toric moment map on a toric manifold is an example of a completely integrable system.

Fix a convex planar polygon $P$ with $n$ sides called the reference polygon. The set of triangulations of $P$ is in natural one-to-one correspondence with the set of trivalent trees with $n$ leaves by sending a triangulation to its dual graph $\Gamma$. We often say a triangulation $\Gamma$ by abuse of notation. The set of triangulations $\Gamma$ can naturally be identified with the set of vertices of the Stasheff associahedron, which in turn is identified with the set of ways to parenthesize a product of $n-1$ elements into binomial operations.

For any triangulation $\Gamma$ of the reference polygon, Kapovich and Millson $[\mathrm{KM}]$ and Klyachko $[\mathrm{Kl}]$ constructed a completely integrable system

$$
\Phi_{\Gamma}: \mathcal{M}_{r} \rightarrow \mathbb{R}^{n-3}
$$


called the bending system. For a particular triangulation $\Gamma$ called the caterpillar (see Figure 1), the bending system comes from the Gelfand-Cetlin system (see $[\mathrm{GS}]$ ) on the Grassmannian $\operatorname{Gr}(2, n)$ through the symplectic reduction (1.2) above (see $[\mathrm{HK}]$ ).

The first main result in this article is the following.

THEOREM 1.1. For any triangulation $\Gamma$ of the reference polygon, there exists a completely integrable system

$$
\Psi_{\Gamma}: \operatorname{Gr}(2, n) \rightarrow \mathbb{R}^{2 n-4}
$$

which induces the bending system $\Phi_{\Gamma}$ on $\mathcal{M}_{\boldsymbol{r}}$ through the symplectic reduction (1.2).

Triangulations of the reference polygon are related to toric degenerations of $\operatorname{Gr}(2, n)$ by Speyer and Sturmfels [SS]. On the other hand, the notion of a toric degeneration of an integrable system is introduced in [NNU1] (see Definition 6.1). Our second main result is the following.

THEOREM 1.2. For any triangulation $\Gamma$ of the reference polygon, the completely integrable system $\Psi_{\Gamma}$ on $\operatorname{Gr}(2, n)$ admits a toric degeneration.

Since the toric degeneration of $\operatorname{Gr}(2, n)$ is $T_{U(n)}$-invariant for any triangulation $\Gamma$, it induces a toric degeneration of $\mathcal{M}_{r}$. The deformation of $\Psi_{\Gamma}$ in Theorem 1.2 is $T_{U(n)}$-invariant, and we obtain the following.

Corollary 1.3. The toric degeneration of $\Psi_{\Gamma}: \operatorname{Gr}(2, n) \rightarrow \mathbb{R}^{2 n-4}$ in Theorem 1.2 induces a toric degeneration of the bending system $\Phi_{\Gamma}: \mathcal{M}_{r} \rightarrow$ $\mathbb{R}^{n-3}$.

Kamiyama and Yoshida [KY] and Howard, Manon, and Millson [HMM] also study the relation between toric degenerations of polygon spaces and bending systems from a slightly different point of view.

For a triangulation $\Gamma$ of the reference polygon, let $X_{\Gamma}$ be the toric variety obtained as the central fiber of the corresponding toric degeneration of $\operatorname{Gr}(2, n)$. The image $\Delta_{\Gamma}$ of the toric moment map of $X_{\Gamma}$ coincides with the moment polytope of the integrable system $\Psi_{\Gamma}: \operatorname{Gr}(2, n) \rightarrow \mathbb{R}^{2 n-4}$ by Theorem 1.2.

THEOREM 1.4. For any pair $\left(\Gamma, \Gamma^{\prime}\right)$ of triangulations of the reference polygon, there is a piecewise-linear automorphism

$$
T_{\Gamma, \Gamma^{\prime}}: \mathbb{R}^{2 n-4} \rightarrow \mathbb{R}^{2 n-4}
$$


of the affine space such that $T_{\Gamma, \Gamma^{\prime}}\left(\Delta_{\Gamma^{\prime}}\right)=\Delta_{\Gamma}$. The map $T_{\Gamma, \Gamma^{\prime}}$ is defined over $\mathbb{Z}$ if $\Delta_{\Gamma}$ is an integral polytope.

If two triangulations are related by a single Whitehead move (see Figure 5), then the corresponding integral piecewise-linear transformation has the form $\left(\ldots, u, u_{1}, u_{2}, u_{3}, u_{4}, \ldots\right) \mapsto\left(\ldots, u^{\prime}, u_{1}, u_{2}, u_{3}, u_{4}, \ldots\right)$, where

$$
\begin{aligned}
u^{\prime}= & u-\min \left(u_{1}+u_{2}, u_{3}+u_{4}\right)+\min \left(u_{1}+u_{4}, u_{2}+u_{3}\right) \\
= & u+\min \left(u_{1}-u_{2}, u_{2}-u_{1}, u_{3}-u_{4}, u_{4}-u_{3}\right) \\
& -\min \left(u_{1}-u_{4}, u_{4}-u_{1}, u_{2}-u_{3}, u_{3}-u_{2}\right)
\end{aligned}
$$

In general, any two triangulations are related by a sequence of Whitehead moves, and the corresponding integral piecewise-linear transformation is an iteration of the transformation above.

The potential function is a Floer-theoretic invariant of Lagrangian submanifolds introduced by Fukaya, Oh, Ohta, and Ono [FO+1]. It gives the "superpotential" of the mirror Landau-Ginzburg model for toric manifolds (see $[\mathrm{CO}],[\mathrm{FO}+2],[\mathrm{FO}+3])$. In [NNU1], the potential function for a Lagrangian torus fiber of the Gelfand-Cetlin system (see [GS]) is computed by using a toric degeneration. An essential point in the argument is the fact that the central fiber is a toric Fano variety admitting a small resolution, which holds also in the present situation, as in the following.

THEOREM 1.5. For any triangulation $\Gamma$ of the reference polygon, the toric variety $X_{\Gamma}$ is a Fano variety admitting a small resolution.

As a result, we obtain an explicit description of the potential function for a Lagrangian torus fiber of the integrable system $\Psi_{\Gamma}$ as a Laurent polynomial over the Novikov ring, as in the following.

THEOREM 1.6. The potential function is given by

$$
\mathfrak{P O}_{\Gamma}=\sum_{\text {triangles }}\left(\frac{y(b) y(c)}{y(a)}+\frac{y(a) y(c)}{y(b)}+\frac{y(a) y(b)}{y(c)}\right),
$$

where $y(a)$ is a Laurent monomial defined in Section 8 associated with an edge a of a triangle, and the sum is taken over all triangles in the triangulation $\Gamma$.

Recall that the geometric lift (see [BZ]) of a piecewise-linear function is given by replacing summation, subtraction, and the minimum by multiplication, division, and summation. 
THEOREM 1.7. For any pair $\left(\Gamma, \Gamma^{\prime}\right)$ of triangulations of the reference polygon, the potential functions $\mathfrak{P O}_{\Gamma}$ and $\mathfrak{P O}_{\Gamma^{\prime}}$ are related by a subtraction-free rational change of variables obtained as the geometric lift of the piecewiselinear transformation $T_{\Gamma, \Gamma^{\prime}}$ in Theorem 1.4.

If $\Gamma$ and $\Gamma^{\prime}$ are related by a single Whitehead move, then the corresponding change of variables is given by

$$
y^{\prime}=y \cdot \frac{y_{1} y_{4}+y_{2} y_{3}}{y_{1} y_{2}+y_{3} y_{4}}=y \cdot \frac{\frac{y_{1}}{y_{2}}+\frac{y_{2}}{y_{1}}+\frac{y_{3}}{y_{4}}+\frac{y_{4}}{y_{3}}+\frac{y_{4}}{y_{1}}+\frac{y_{2}}{y_{3}}+\frac{y_{3}}{y_{2}}}{,}
$$

which indeed is a geometric lift of (1.3).

On the other hand, the central fiber of the toric degeneration of a polygon space may neither be Fano nor admit a small resolution, and one cannot apply the argument of [NNU1] directly to this case. For example, the moduli space of pentagons is isomorphic to $\mathbb{C P}^{1} \times \mathbb{C P}^{1}$ for a suitable choice of $\boldsymbol{r}$, and it degenerates to the Hirzebruch surface of degree 2, which is not Fano. In this case, we need to consider contributions of sphere bubbles to the potential function. This case is studied in detail by Auroux [A1], [A2] and by Fukaya, Oh, Ohta, and Ono $[\mathrm{FO}+4]$.

Our article has the following organization. In Section 2, we recall basic facts on polygon spaces. We study the bending systems in Section 3. Theorem 1.1 is proved in Section 4, and Theorem 1.4 is a corollary to Proposition 4.10. Section 5 is devoted to a construction of toric degenerations of $\operatorname{Gr}(2, n)$ in stages, which are used to construct a deformation of completely integrable systems. Theorem 1.2 is proved in Section 6, and Theorem 1.5 is proved in Section 7. In Section 8, we compute the potential functions for the completely integrable systems on $\operatorname{Gr}(2, n)$, and we prove Theorems 1.6 and 1.7 .

\section{§. Polygon spaces and Grassmannians}

In this section, we fix notation and recall the relation between polygon spaces and Grassmannians.

We identify the Lie algebra $\mathfrak{u}(m)$ (resp., $\mathfrak{s u}(m)$ ) of the Lie algebra of the unitary group $U(m)$ (resp., the special unitary group $\mathrm{SU}(m)$ ) with the space $\sqrt{-1} \mathfrak{u}(m)$ of Hermitian matrices (resp., the space $\sqrt{-1} \mathfrak{s u}(m)$ of traceless Hermitian matrices). The dual space $\mathfrak{u}(m)^{*}$ (resp., $\left.\mathfrak{s u}(m)^{*}\right)$ is identified with $\sqrt{-1} \mathfrak{u}(m)$ (resp., $\sqrt{-1} \mathfrak{s} \mathfrak{u}(m))$ by the invariant inner product $\langle x, y\rangle=\operatorname{tr}(x y)$. The moment map of the natural $U(m)$-action on $\mathbb{C}^{m}$ equipped with the 
standard symplectic structure is given by

$$
\mathbb{C}^{m} \longrightarrow \sqrt{-1} \mathfrak{u}(m), \quad z=\left(\begin{array}{c}
z_{1} \\
\vdots \\
z_{m}
\end{array}\right) \longmapsto \frac{1}{2} z z^{*}=\frac{1}{2}\left(z_{i} \bar{z}_{j}\right)_{i, j}
$$

Recall from Section 1 that the polygon space $\mathcal{M}_{\boldsymbol{r}}$ is defined by

$$
\mathcal{M}_{r}=\left\{\boldsymbol{x}=\left(x_{1}, \ldots, x_{n}\right) \in \prod_{i=1}^{n} S^{2}\left(r_{i}\right) \mid x_{1}+\cdots+x_{n}=0\right\} / \mathrm{SO}(3)
$$

where $n \geq 3$ and $r_{i}<r_{1}+\cdots+r_{i-1}+r_{i+1}+\cdots+r_{n}$ for $i=1, \ldots, n$. To describe a natural symplectic structure on $\mathcal{M}_{\boldsymbol{r}}$, we identify $\mathbb{R}^{3}$ with $\sqrt{-1} \mathfrak{s u}(2)$ by

$$
\begin{aligned}
h: & \mathbb{R}^{3} \stackrel{\sim}{\rightarrow} \\
\Psi & \stackrel{\sqrt{-1} \mathfrak{s u}(2)}{\Psi} \\
\left(\begin{array}{l}
x^{1} \\
x^{2} \\
x^{3}
\end{array}\right) & \mapsto\left(\begin{array}{cc}
x^{3} & x^{1}-\sqrt{-1} x^{2} \\
x^{1}+\sqrt{-1} x^{2} & -x^{3}
\end{array}\right) .
\end{aligned}
$$

Then the $\mathrm{SO}(3)$-action on $\mathbb{R}^{3}$ is induced from the (co)adjoint action of $\mathrm{SU}(2)$, and the sphere $S^{2}\left(r_{i}\right)$ is identified with a (co)adjoint orbit $\mathcal{O}_{r_{i}}$ of $\operatorname{diag}\left(r_{i},-r_{i}\right)$, which has the canonical Kostant-Kirillov symplectic form $\omega_{\mathcal{O}_{r_{i}}}$. We equip $\mathcal{O}_{r_{1}} \times \cdots \times \mathcal{O}_{r_{n}}$ with the symplectic form $\sum_{i} \operatorname{pr}_{i}^{*} \omega_{\mathcal{O}_{r_{i}}}$, where $\operatorname{pr}_{i}: \mathcal{O}_{r_{1}} \times \cdots \times \mathcal{O}_{r_{n}} \rightarrow \mathcal{O}_{r_{i}}$ is the $i$ th projection. Then the diagonal $\mathrm{SU}(2)-$ action is Hamiltonian, and its moment map is given by

$$
\mu: \mathcal{O}_{r_{1}} \times \cdots \times \mathcal{O}_{r_{n}} \longrightarrow \sqrt{-1} \mathfrak{s u}(2)^{*}, \quad\left(x_{1}, \ldots, x_{n}\right) \longmapsto x_{1}+\cdots+x_{n} .
$$

Hence, one has the following.

Proposition 2.1. The polygon space $\mathcal{M}_{r}$ is a symplectic reduction of $\mathcal{O}_{r_{1}} \times \cdots \times \mathcal{O}_{r_{n}}$ by the diagonal $\mathrm{SU}(2)$-action

$$
\mathcal{M}_{\boldsymbol{r}}=\prod_{i=1}^{n} \mathcal{O}_{r_{i}} / /_{0} \mathrm{SU}(2)=\mu^{-1}(0) / \mathrm{SU}(2)
$$

Let $\omega_{\mathcal{M}_{r}}$ denote the induced symplectic form on $\mathcal{M}_{\boldsymbol{r}}$. Identifying the symplectic reduction with a GIT quotient $\left(\prod_{i=1}^{n} \mathbb{C P}^{1}\right) / / \mathrm{SL}(2, \mathbb{C})$, we obtain a compatible complex structure on $\left(\mathcal{M}_{r}, \omega_{\mathcal{M}_{r}}\right)$. 
Next we recall a relation to the Grassmannian $\operatorname{Gr}(2, n)$. Let $|\boldsymbol{r}|=\sum_{i} r_{i}$. We consider the natural right $U(2)$-action on the vector space $\mathbb{C}^{n \times 2}$ of $n \times 2$ matrices. From (2.1), its moment map is given by

$$
\text { (2.3) } \mu_{U(2)}: \mathbb{C}^{n \times 2} \rightarrow \sqrt{-1} \mathfrak{u}(2), \quad\left(\begin{array}{cc}
z_{1} & w_{1} \\
\vdots & \vdots \\
z_{n} & w_{n}
\end{array}\right) \longmapsto \frac{1}{2} \sum_{i=1}^{n}\left(\begin{array}{cc}
\left|z_{i}\right|^{2} & \bar{z}_{i} w_{i} \\
z_{i} \bar{w}_{i} & \left|w_{i}\right|^{2}
\end{array}\right) .
$$

Then

$$
\left(\begin{array}{cc}
z_{1} & w_{1} \\
\vdots & \vdots \\
z_{n} & w_{n}
\end{array}\right) \in \mu_{U(2)}^{-1}\left(\begin{array}{cc}
|\boldsymbol{r}| & 0 \\
0 & |\boldsymbol{r}|
\end{array}\right)
$$

if and only if it satisfies

$$
\sum_{i}\left|z_{i}\right|^{2}=\sum_{i}\left|w_{i}\right|^{2}=2|\boldsymbol{r}|, \quad \sum_{i} z_{i} \bar{w}_{i}=0 .
$$

It follows that $\operatorname{Gr}(2, n)$ is a symplectic reduction of $\mathbb{C}^{n \times 2}$ by the $U(2)$-action

$$
\operatorname{Gr}(2, n)=\mathbb{C}^{n \times 2} / /|\boldsymbol{r}| U(2)=\mu_{U(2)}^{-1}\left(\begin{array}{cc}
|\boldsymbol{r}| & 0 \\
0 & |\boldsymbol{r}|
\end{array}\right) / U(2) .
$$

We consider the moment map

$$
\mu_{\mathrm{SU}(2)}: \mathbb{C}^{2} \longrightarrow \sqrt{-1} \mathfrak{s u}(2), \quad(z, w) \longmapsto \frac{1}{4}\left(\begin{array}{cc}
|z|^{2}-|w|^{2} & 2 \bar{z} w \\
2 z \bar{w} & |w|^{2}-|z|^{2}
\end{array}\right)
$$

of the standard $\mathrm{SU}(2)$-action on $\mathbb{C}^{2}$. Condition (2.4) implies that

$$
\left(\mu_{\mathrm{SU}(2)}\left(z_{1}, w_{1}\right), \ldots, \mu_{\mathrm{SU}(2)}\left(z_{n}, w_{n}\right)\right)
$$

gives a closed $n$-gon in $\sqrt{-1} \mathfrak{s u}(2) \cong \mathbb{R}^{3}$ (i.e., it satisfies $\sum_{i} \mu_{\mathrm{SU}(2)}\left(z_{i}, w_{i}\right)=$ $0)$. Since $\mu_{\mathrm{SU}(2)}: \mathbb{C}^{2} \rightarrow \sqrt{-1} \mathfrak{s u}(2)$ is a quotient map by the diagonal $S^{1}$ action on $\mathbb{C}^{2}$, the quotient $T_{U(n)} \backslash \operatorname{Gr}(2, n)$ can be regarded as a moduli space of polygons with fixed perimeter $|\boldsymbol{r}|$, where $T_{U(n)} \subset U(n)$ is the maximal torus consisting of diagonal matrices. Note that the $U(n)$-action on $\operatorname{Gr}(2, n)$ has the stabilizer of positive dimension. The moment map $\mu_{T_{U(n)}}$ : $\operatorname{Gr}(2, n) \rightarrow \mathbb{R}^{n}$ of the $T_{U(n)}$-action on $\operatorname{Gr}(2, n)$ is given by

$$
\mu_{T_{U(n)}}:\left[\begin{array}{cc}
z_{1} & w_{1} \\
\vdots & \vdots \\
z_{n} & w_{n}
\end{array}\right] \longmapsto\left(\frac{\left|z_{1}\right|^{2}+\left|w_{1}\right|^{2}}{2}, \ldots, \frac{\left|z_{n}\right|^{2}+\left|w_{n}\right|^{2}}{2}\right) .
$$

Since $\left(\left|z_{i}\right|^{2}+\left|w_{i}\right|^{2}\right) / 2=2\left\|h^{-1} \circ \mu_{\mathrm{SU}(2)}\left(z_{i}, w_{i}\right)\right\|_{\mathbb{R}^{3}}$, we have the following. 
Proposition 2.2 ([HK, (3.9)]). The polygon space $\mathcal{M}_{\boldsymbol{r}}$ is isomorphic to the symplectic reduction of $\operatorname{Gr}(2, n)$ by the $T_{U(n)}$-action

$$
\mathcal{M}_{\boldsymbol{r}} \cong T_{U(n)} \backslash_{2 \boldsymbol{r}} \operatorname{Gr}(2, n)=T_{U(n)} \backslash \mu_{T}^{-1}(2 \boldsymbol{r}),
$$

where the symplectic structure on $\operatorname{Gr}(2, n)$ is the Kostant-Kirillov form on the (co)adjoint orbit of $\operatorname{diag}(|\boldsymbol{r}|,|\boldsymbol{r}|, 0, \ldots, 0)$ in $\sqrt{-1} \mathfrak{u}(n)\left(\cong \mathfrak{u}(n)^{*}\right)$.

Propositions 2.1 and 2.2 can be summarized as

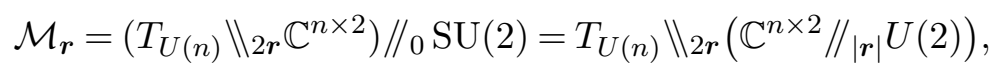

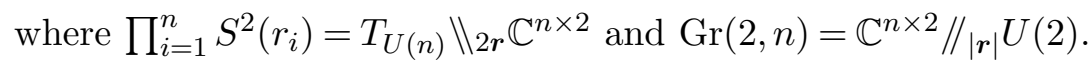

\section{$\S 3 . \quad$ Bending Hamiltonians}

Fix a convex $n$-gon $P \subset \mathbb{R}^{2}$, and call it the reference $n$-gon. Let $e_{1}, \ldots, e_{n}$ denote the sides of $P$ labeled in cyclic order. For an oriented diagonal $d$ of $P$, we write the corresponding diagonal of $\boldsymbol{x} \in \mathcal{M}_{\boldsymbol{r}}$ as $d(\boldsymbol{x})$. If $d$ connects the $i$ th vertex and the $j$ th vertex for $i<j$, then $d(\boldsymbol{x})$ is given by

$$
d(\boldsymbol{x})=x_{i+1}+x_{i+2}+\cdots+x_{j}
$$

or

$$
d(\boldsymbol{x})=x_{j+1}+\cdots+x_{n}+x_{1}+\cdots+x_{i},
$$

depending on the orientation of $d$. We define $\varphi_{d}: \mathcal{M}_{r} \rightarrow \mathbb{R}$ to be the length function

$$
\varphi_{d}(\boldsymbol{x})=|d(\boldsymbol{x})|
$$

of the diagonal. Kapovich and Millson $[\mathrm{KM}]$ proved that its Hamiltonian flow folds the polygon along the diagonal $d$ at a constant speed. Thus, $\varphi_{d}$ is called a bending Hamiltonian.

We say that two diagonals $d$ and $d^{\prime}$ are noncrossing if they do not intersect in the interior of $P$.

Theorem 3.1 ([KM, Section 3], [Kl, Section 2.1]). If diagonals $d$ and $d^{\prime}$ are noncrossing, then $\varphi_{d}$ and $\varphi_{d^{\prime}}$ are Poisson commutative. Furthermore, each choice of $(n-3)$ mutually noncrossing diagonals gives a completely integrable system on $\mathcal{M}_{\boldsymbol{r}}$, and the functions $\varphi_{d}$ give action variables. 


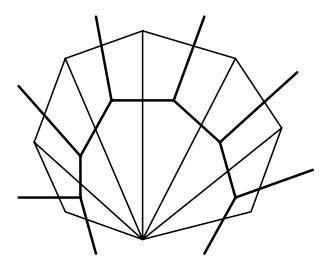

Figure 1: The caterpillar.

Note that such a choice of $(n-3)$ diagonals defines a triangulation of the reference $n$-gon $P$. Here we consider only triangulations whose vertices coincide with those of $P$. Let $\Gamma$ denote the dual graph of a given triangulation. The graph $\Gamma$ is a trivalent tree with $n$ leaves labeled by sides $e_{1}, \ldots, e_{n}$ of $P$ and $(n-3)$ interior edges labeled by the diagonals $d_{1}, \ldots, d_{n-3}$. We often say a triangulation $\Gamma$ by abuse of notation. We write the completely integrable system given by $\Gamma$ as

$$
\Phi_{\Gamma}=\left(\varphi_{d_{1}}, \ldots, \varphi_{d_{n-3}}\right): \mathcal{M}_{r} \longrightarrow \mathbb{R}^{n-3},
$$

and we call it the bending system associated to $\Gamma$. The image

$$
\Delta_{\Gamma}(\boldsymbol{r}):=\Phi_{\Gamma}\left(\mathcal{M}_{\boldsymbol{r}}\right) \subset \mathbb{R}^{n-3}
$$

is the convex polytope defined by triangle inequalities.

EXAMPLE 3.2. The triangulation given by $d_{\alpha}=e_{1}+\cdots+e_{\alpha+1}(\alpha=$ $1, \ldots, n-3)$ is called the caterpillar (see Figure 1$)$. Let $\left(u_{1}, \ldots, u_{n-3}\right)$ be the coordinates on $\mathbb{R}^{n-3}$ corresponding to the bending Hamiltonians $\varphi_{d_{\alpha}}$. The image $\Delta_{\Gamma}(\boldsymbol{r})$ is a polytope defined by triangle inequalities

$$
\begin{aligned}
& \left|r_{1}-r_{2}\right| \leq u_{1} \leq r_{1}+r_{2}, \\
& \left|u_{1}-r_{3}\right| \leq u_{2} \leq u_{1}+r_{3},
\end{aligned}
$$

$$
\begin{gathered}
\left|u_{n-4}-r_{n-2}\right| \leq u_{n-3} \leq u_{n-4}+r_{n-2}, \\
\left|r_{n-1}-r_{n}\right| \leq u_{n-3} \leq r_{n-1}+r_{n} .
\end{gathered}
$$

EXAMPLE 3.3. Suppose that $n=5$, and consider a triangulation given by $d_{1}=e_{1}+e_{2}, d_{2}=e_{1}+e_{2}+e_{3}$. If all side lengths $r_{1}, \ldots, r_{4}$ are close, then $\mathcal{M}_{r}$ is isomorphic to $\mathbb{C P}^{2}$ blown up at four distinct points (see [Kl, Example 10.4] or [HK, (6.3)]), and the image $\Delta_{\Gamma}(\boldsymbol{r})$ is a heptagon shown in Figure 2. 


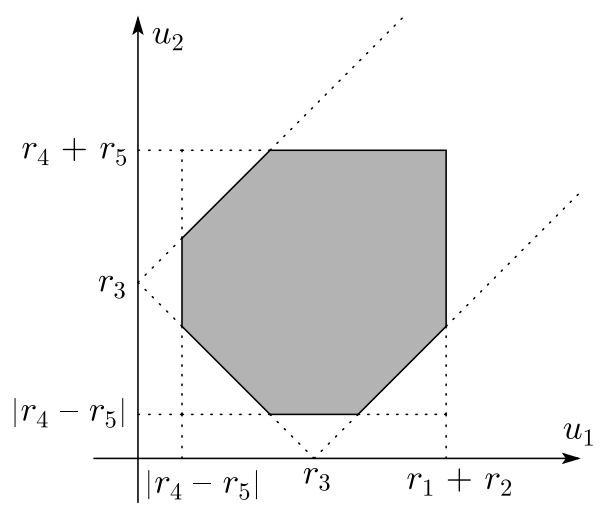

Figure 2: The image of the bending system in Example 3.3.
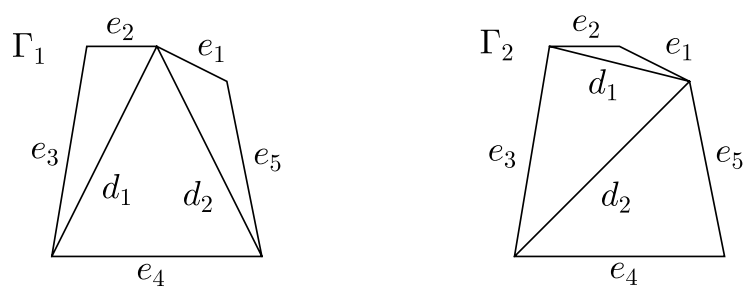

Figure 3: Two triangulations in Example 3.4.

EXAMPLE 3.4. Let $n=5$, and assume that $r_{1}>r_{2}>0$ and that

$$
r_{1}+r_{2}<\min \left(-r_{3}+r_{4}+r_{5}, r_{3}-r_{4}+r_{5}, r_{3}+r_{4}-r_{5}\right) .
$$

In this case, $\mathcal{M}_{r}$ is isomorphic to $\mathbb{C P}^{1} \times \mathbb{C P}^{1}$ (see $[\mathrm{HK},(6.2)]$ or $[$ Fo, Section 5]). We consider two triangulations $\Gamma_{1}$ and $\Gamma_{2}$ shown in Figure 3. The images $\Delta_{\Gamma_{1}}(\mathbf{r})$ and $\Delta_{\Gamma_{2}}(\boldsymbol{r})$ of the corresponding bending systems are shown in Figure 4. Note that $\Delta_{\Gamma_{1}}(\boldsymbol{r})$ is the moment polytope of the standard moment map on $\mathbb{C P}^{1} \times \mathbb{C P}^{1}$, while $\Delta_{\Gamma_{2}}(\boldsymbol{r})$ is that of the Hirzebruch surface $F_{2}=\mathbb{P}\left(\mathcal{O}_{\mathbb{P}^{1}} \oplus \mathcal{O}_{\mathbb{P}^{1}}(2)\right)$ of degree 2 . Note that $F_{2}$ is symplectomorphic to $\mathbb{C P}^{1} \times \mathbb{C P}^{1}$ (but not isomorphic as complex manifolds).

As we have seen in Example 3.4, the bending system, and hence the corresponding image $\Delta_{\Gamma}(\boldsymbol{r})$, depends on the choice of a triangulation $\Gamma$. We compare the polytopes $\Delta_{\Gamma_{i}}(\boldsymbol{r})$ for different triangulations $\Gamma_{1}$ and $\Gamma_{2}$. Recall that a Whitehead move (for triangulations) replaces a diagonal of a 

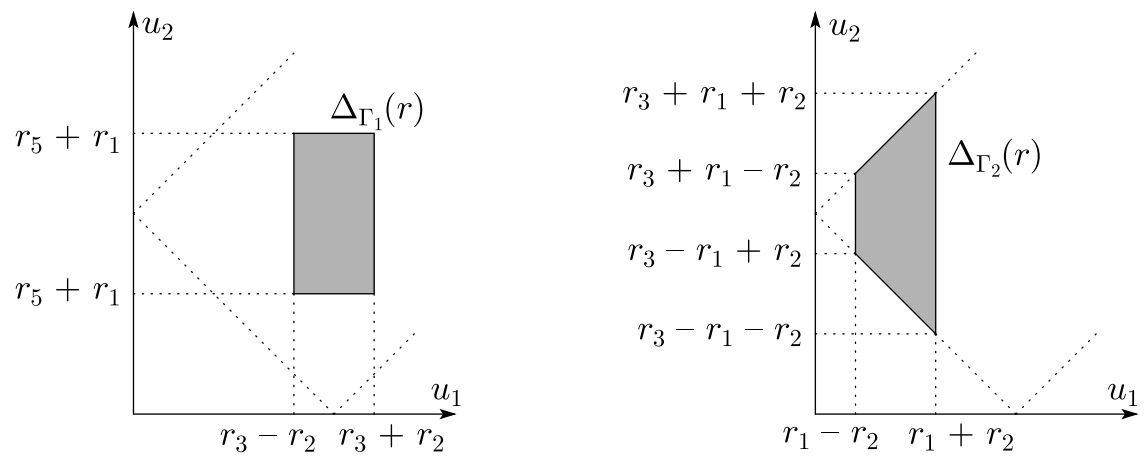

Figure 4: The images $\Delta_{\Gamma_{1}}(\boldsymbol{r})$ and $\Delta_{\Gamma_{2}}(\boldsymbol{r})$ in Example 3.4.

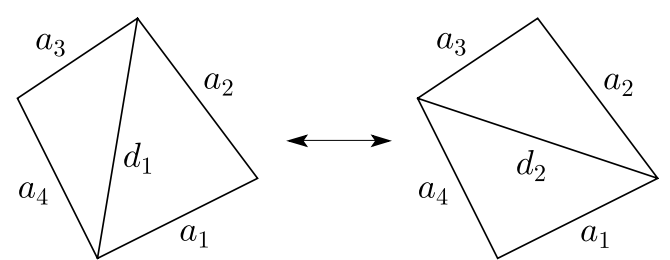

Figure 5: A Whitehead move.

quadrilateral with the other one (see Figure 5). Since triangulations can be transformed into each other by sequences of Whitehead moves, it suffices to consider the case where $\Gamma_{1}$ and $\Gamma_{2}$ can be transformed to each other by a single Whitehead move. Suppose that $\Gamma_{1}$ is transformed into $\Gamma_{2}$ by a Whitehead move in a quadrilateral with sides $a_{1}, a_{2}, a_{3}, a_{4}$ replacing a diagonal $d= \pm\left(a_{1}+a_{2}\right)$ with $d^{\prime}= \pm\left(a_{2}+a_{3}\right)$. Note that $a_{i}$ is either a side $e_{j}$ of $P$ or a diagonal $d_{\alpha}$ contained in both of $\Gamma_{1}$ and $\Gamma_{2}$. Let $u, u^{\prime}$, and $u_{i}$ be the coordinates corresponding to $d, d^{\prime}$, and $a_{i}$, respectively, where we assume that $u_{i}=r_{j}$ is a constant if $a_{i}$ is a side $e_{j}$ of $P$.

Proposition 3.5. Under the above situation, the piecewise-linear transformation

$$
\begin{aligned}
u^{\prime}= & u-\min \left(u_{1}+u_{2}, u_{3}+u_{4}\right)+\min \left(u_{1}+u_{4}, u_{2}+u_{3}\right) \\
= & u+\min \left(u_{1}-u_{2}, u_{2}-u_{1}, u_{3}-u_{4}, u_{4}-u_{3}\right) \\
& -\min \left(u_{1}-u_{4}, u_{4}-u_{1}, u_{2}-u_{3}, u_{3}-u_{2}\right)
\end{aligned}
$$


gives a bijection $\Delta_{\Gamma_{1}}(\boldsymbol{r}) \rightarrow \Delta_{\Gamma_{2}}(\boldsymbol{r})$. In particular, this map preserves the area of $\Delta_{\Gamma_{i}}(\boldsymbol{r})$ and the number of integral points in $\Delta_{\Gamma_{i}}(\boldsymbol{r})$ in the case where $\Delta_{\Gamma_{i}}(\boldsymbol{r})$ is an integral polytope (i.e., $\left.\boldsymbol{r} \in\left(\mathbb{Z}_{>0}\right)^{n}\right)$.

Proof. Since the triangle inequalities for $u$ are

$$
\max \left(\left|u_{1}-u_{2}\right|,\left|u_{3}-u_{4}\right|\right) \leq u \leq \min \left(u_{1}+u_{2}, u_{3}+u_{4}\right),
$$

the length of the range of $u$ for fixed $u_{1}, \ldots, u_{4}$ is

$$
\min \left(u_{1}+u_{2}, u_{3}+u_{4}\right)-\max \left(\left|u_{1}-u_{2}\right|,\left|u_{3}-u_{4}\right|\right) .
$$

Since $\min \left\{a_{i} \mid i \in I\right\}+\min \left\{b_{j} \mid j \in J\right\}=\min \left\{a_{i}+b_{j} \mid i \in I, j \in J\right\}$, we have

$$
\begin{aligned}
& \min \left(u_{1}+u_{2}, u_{3}+u_{4}\right)-\max \left(\left|u_{1}-u_{2}\right|,\left|u_{3}-u_{4}\right|\right) \\
& \quad=\min \left(u_{1}+u_{2}, u_{3}+u_{4}\right)+\min \left(u_{1}-u_{2}, u_{2}-u_{1}, u_{3}-u_{4}, u_{4}-u_{3}\right) \\
& \quad=\min \left\{2 u_{i}, u_{1}+u_{2}+u_{3}+u_{4}-2 u_{j} \mid 1 \leq i, j \leq 4\right\} .
\end{aligned}
$$

Similarly, the length of the range of $u^{\prime}$ is

$$
\begin{aligned}
& \min \left(u_{1}+u_{4}, u_{2}+u_{3}\right)+\min \left(u_{1}-u_{4}, u_{4}-u_{1}, u_{2}-u_{3}, u_{3}-u_{2}\right) \\
& \quad=\min \left\{2 u_{i}, u_{1}+u_{2}+u_{3}+u_{4}-2 u_{j} \mid 1 \leq i, j \leq 4\right\}
\end{aligned}
$$

which is identical to (3.3). Hence, (3.2) gives an area-preserving transformation. Moreover, if $u_{1}, \ldots, u_{4} \in \mathbb{Z}$, then (3.2) is defined over $\mathbb{Z}$, and hence the number of integral $u$ is also preserved.

ExAmple 3.6. Let $\Gamma_{1}$ and $\Gamma_{2}$ be the triangulation in Example 3.4. Now $\Gamma_{1}$ can be transformed into $\Gamma_{2}$ by a two-step Whitehead move shown in Figure 6 . Let $u_{\alpha}$ and $u_{\alpha}^{\prime}(\alpha=1,2)$ be the coordinates corresponding to $d_{\alpha}$

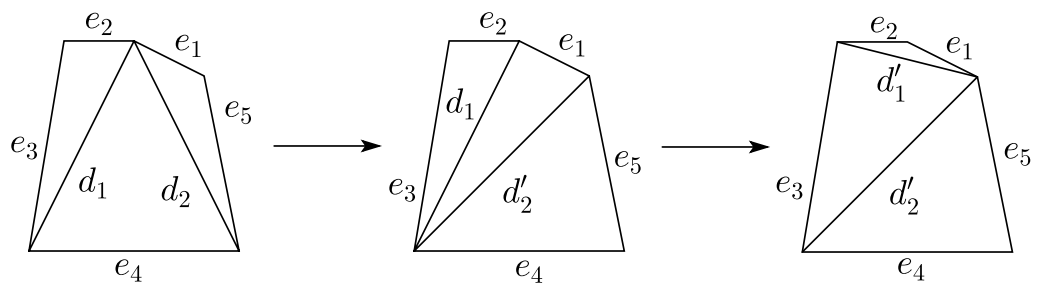

Figure 6: Whitehead moves sending $\Gamma_{1}$ to $\Gamma_{2}$. 
and $d_{\alpha}^{\prime}$, respectively. The piecewise-linear map corresponding to the first Whitehead move is given by

$$
\left(u_{1}, u_{2}^{\prime}\right)=\left(u_{1}, u_{2}+\max \left(\left|u_{1}-r_{1}\right|,\left|r_{4}-r_{5}\right|\right)-\max \left(\left|u_{1}-r_{4}\right|, r_{5}-r_{1}\right)\right) .
$$

Since

$$
\max \left(\left|u_{1}-r_{1}\right|,\left|r_{4}-r_{5}\right|\right)=u_{1}-r_{1}, \quad \max \left(\left|u_{1}-r_{4}\right|, r_{5}-r_{1}\right)=r_{5}-r_{1}
$$

on $r_{3}-r_{2}<u_{1}<r_{3}+r_{2}$, the above map is given by

$$
\left(u_{1}, u_{2}^{\prime}\right)=\left(u_{1}, u_{2}+u_{1}-r_{5}\right)
$$

on $\Delta_{\Gamma_{1}}(\boldsymbol{r})$ (see Figure 7 ).
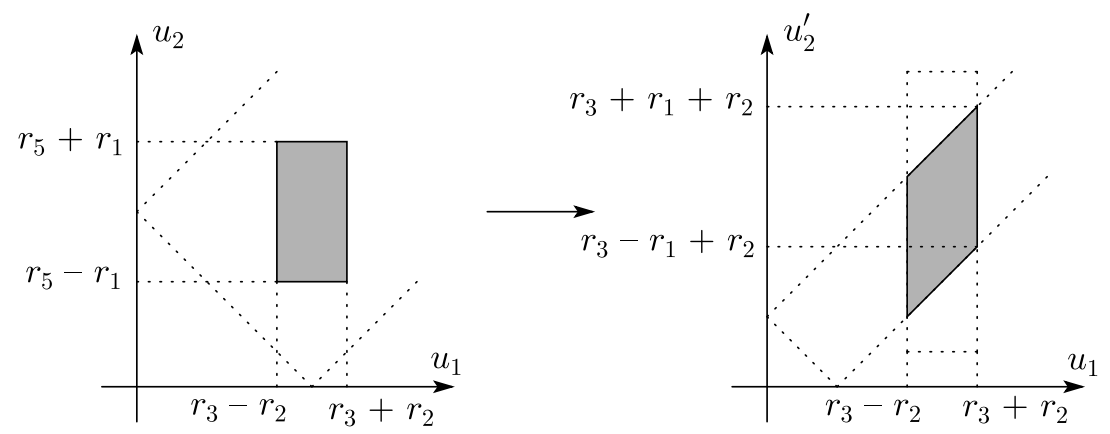

Figure 7: The first piecewise-linear transformation in Example 3.6.

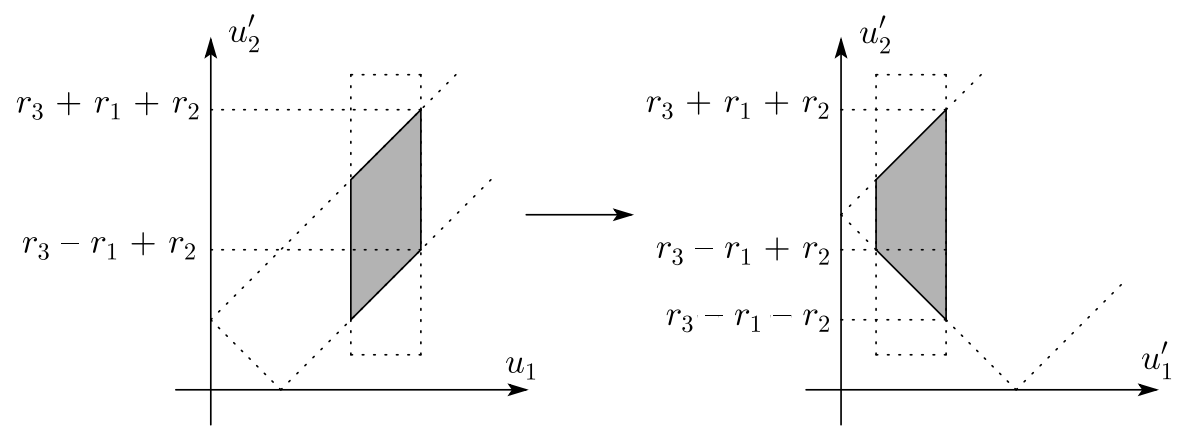

Figure 8: The second piecewise-linear transformation in Example 3.6. 
The piecewise-linear transformation for the second Whitehead move is

$$
\left(u_{1}^{\prime}, u_{2}^{\prime}\right)=\left(u_{1}+\max \left(\left|u_{2}^{\prime}-r_{3}\right|,\left(r_{1}-r_{2}\right)\right)-\max \left(\left|u_{2}^{\prime}-r_{1}\right|, r_{3}-r_{2}\right), u_{2}^{\prime}\right),
$$

which coincides with

$$
\left(u_{1}^{\prime}, u_{2}^{\prime}\right)=\left(u_{1}-\min \left(u_{2}^{\prime}-r_{2}, r_{3}-r_{1}\right), u_{2}^{\prime}\right)
$$

on the image of $\Delta_{\Gamma_{1}}(\boldsymbol{r})$ (see Figure 8).

\section{§4. Completely integrable systems on $\operatorname{Gr}(2, n)$}

Hausmann and Knutson [HK] proved that the Gelfand-Cetlin system (see [GS]) on $\operatorname{Gr}(2, n)$ induces the bending system on $\mathcal{M}_{r}$ under the symplectic reduction in the case where the triangulation is the caterpillar. In this section, we construct a completely integrable system on $\operatorname{Gr}(2, n)$ that induces the bending systems for each triangulation $\Gamma$.

Fix a triangulation $\Gamma$ of the reference $n$-gon $P$. For each side $e_{i}(i=$ $1, \ldots, n)$ and diagonal $d_{\alpha}(\alpha=1, \ldots, n-3)$, we associate a subgroup of $U(n)$ as follows. For a side $e_{i}$, we associate a subgroup isomorphic to $S^{1}$ given by

$$
S_{i}^{1}=S_{e_{i}}^{1}=\left(\begin{array}{ccc}
1_{i-1} & & \\
& S^{1} & \\
& & 1_{n-i}
\end{array}\right) \subset U(n) .
$$

For a diagonal $d_{\alpha}=\sum_{i \in I_{\alpha}} e_{i}$, the corresponding subgroup $U_{\alpha}=U_{d_{\alpha}} \cong U\left(\left|I_{\alpha}\right|\right)$ is defined by

$$
U_{d_{\alpha}}=\left\{\left(g_{i j}\right) \in U(n) \mid\left(g_{i j}\right)_{i, j \in I_{\alpha}} \in U\left(\left|I_{\alpha}\right|\right) \text { and } g_{i j}=\delta_{i j} \text { for }(i, j) \notin I_{\alpha}^{2}\right\} \text {. }
$$

REMARK 4.1. The noncrossing condition for $d_{\alpha}, d_{\beta}$ is equivalent to that $I_{\alpha}$ and $I_{\beta}$ satisfy $I_{\alpha} \supset I_{\beta}, I_{\alpha} \subset I_{\beta}$, or $I_{\alpha} \cap I_{\beta}=\emptyset$. Hence, each pair $G_{1}, G_{2} \in$ $\left\{U_{1}, \ldots, U_{n-3}, S_{1}^{1}, \ldots, S_{n}^{1}\right\}$ of subgroups satisfies $G_{1} \subset G_{2}, G_{1} \supset G_{2}$, or $G_{1} \cap$ $G_{2}=\{1\}$ in $U(n)$.

Recall that the moment map $\mu_{U(n)}: \operatorname{Gr}(2, n) \rightarrow \mathfrak{u}(n)^{*} \cong \sqrt{-1} \mathfrak{u}(n)$ of the $U(n)$-action is given by

$$
\left[\begin{array}{cc}
z_{1} & w_{1} \\
\vdots & \vdots \\
z_{n} & w_{n}
\end{array}\right] \longmapsto \frac{1}{2}\left(\begin{array}{cc}
z_{1} & w_{1} \\
\vdots & \vdots \\
z_{n} & w_{n}
\end{array}\right)\left(\begin{array}{ccc}
\bar{z}_{1} & \ldots & \bar{z}_{n} \\
\bar{w}_{1} & \ldots & \bar{w}_{n}
\end{array}\right)=\left(\frac{z_{i} \bar{z}_{j}+w_{i} \bar{w}_{j}}{2}\right)_{i, j}
$$


Then the moment maps for $S_{e_{i}}^{1}$ and $U_{d_{\alpha}}$ are given by

$$
\psi_{e_{i}}: \operatorname{Gr}(2, n) \longrightarrow \mathbb{R}, \quad \psi_{e_{i}}(z, w)=\frac{\left|z_{i}\right|^{2}+\left|w_{i}\right|^{2}}{2}
$$

and

$$
\mu_{U_{\alpha}}: \operatorname{Gr}(2, n) \rightarrow \sqrt{-1} \mathfrak{u}\left(\left|I_{\alpha}\right|\right), \quad \mu_{U_{\alpha}}(z, w)=\left(\frac{z_{i} \bar{z}_{j}+w_{i} \bar{w}_{j}}{2}\right)_{i, j \in I_{\alpha}},
$$

respectively. Note that $\mu_{U(n)}$ embeds $\operatorname{Gr}(2, n)$ into $\sqrt{-1} \mathfrak{u}(n)$ as the adjoint orbit of $\operatorname{diag}(|\boldsymbol{r}|,|\boldsymbol{r}|, 0, \ldots, 0)$. In particular, $\mu_{U(n)}(z, w)$ has rank 2 for any $[z, w] \in \operatorname{Gr}(2, n)$, and hence the rank of $\mu_{U_{\alpha}}(z, w)$ is at most 2 . Let $\lambda_{\alpha, 1}(z, w) \geq$ $\lambda_{\alpha, 2}(z, w) \geq 0$ be the first and second eigenvalues of $\mu_{U_{\alpha}}(z, w)$.

Recall that the moment map is a Poisson morphism, as in the following.

Proposition $4.2([\mathrm{Ko}])$. Let $G$ be a compact Lie group acting on a symplectic manifold $(X, \omega)$ with a moment map $\mu: X \rightarrow \mathfrak{g}^{*}$. For $f_{1}, f_{2} \in C^{\infty}\left(\mathfrak{g}^{*}\right)$, we have

$$
\left\{\mu^{*} f_{1}, \mu^{*} f_{2}\right\}_{X}=\mu^{*}\left\{f_{1}, f_{2}\right\}_{\mathfrak{g}^{*}},
$$

where $\{,\}_{\mathfrak{g}^{*}}$ is the natural Poisson structure on $\mathfrak{g}^{*}$.

This immediately yields the following.

COROLlary 4.3. Let $G$ be a compact Lie group acting on a symplectic manifold $(X, \omega)$ with a moment map $\mu: X \rightarrow \mathfrak{g}^{*}$, and let $f_{1}, f_{2} \in C^{\infty}\left(\mathfrak{g}^{*}\right)$ be smooth functions on $\mathfrak{g}^{*}$. If either $f_{1}$ or $f_{2}$ is $\operatorname{Ad}^{*}(G)$-invariant (i.e., if it is constant on each coadjoint orbit), then $\mu^{*} f_{1}$ and $\mu^{*} f_{2}$ are Poisson commutative:

$$
\left\{\mu^{*} f_{1}, \mu^{*} f_{2}\right\}_{X}=0
$$

We also have the following.

Lemma 4.4. Let $(X, \omega)$ be a symplectic manifold, and assume that two Lie groups $G_{1}, G_{2}$ act on $(X, \omega)$ in Hamiltonian fashion with moment maps $\mu_{i}: X \rightarrow \mathfrak{g}_{i}^{*}, i=1,2$. If $G_{1}$ and $G_{2}$ satisfy either $G_{1} \subset G_{2}$ or $G_{1} \cap G_{2}=\{1\}$ in the group of symplectomorphisms, then

$$
\left\{\mu_{1}^{*} f_{1}, \mu_{2}^{*} f_{2}\right\}_{X}=0
$$

for $\operatorname{Ad}^{*}\left(G_{i}\right)$-invariant functions $f_{i} \in C^{\infty}\left(\mathfrak{g}_{i}^{*}\right)^{G_{i}}, i=1,2$. 
Proof. We first assume that $G_{1} \subset G_{2}$. Then $\mu_{1}$ is a composition of $\mu_{2}$ : $X \rightarrow \mathfrak{g}_{2}^{*}$ and the natural projection $p: \mathfrak{g}_{2}^{*} \rightarrow \mathfrak{g}_{1}^{*}$. From Proposition 4.2, we have

$$
\left\{\mu_{1}^{*} f_{1}, \mu_{2}^{*} f_{2}\right\}_{X}=\left\{\mu_{2}^{*} p^{*} f_{1}, \mu_{2}^{*} f_{2}\right\}_{X}=\mu_{2}^{*}\left\{p^{*} f_{1}, f_{2}\right\}_{\mathfrak{g}_{2}^{*}}=0 .
$$

Next, we consider the second case. Then the moment map of the action of $G=G_{1} \times G_{2}$ is given by

$$
\mu=\left(\mu_{1}, \mu_{2}\right): X \longrightarrow \mathfrak{g}_{1}^{*} \oplus \mathfrak{g}_{2}^{*}
$$

and we have $\mu_{i}=p_{i} \circ \mu$, where $p_{i}: \mathfrak{g}_{1}^{*} \oplus \mathfrak{g}_{2}^{*} \rightarrow \mathfrak{g}_{i}^{*}$ is the $i$ th projection. Since $\mu_{i}^{*} f_{i}=\mu^{*}\left(p_{i}^{*} f_{i}\right)$ and $p_{i}^{*} f_{i} \in C^{\infty}\left(\mathfrak{g}_{1}^{*} \oplus \mathfrak{g}_{2}^{*}\right)^{G}$, the Poisson commutativity follows from the argument in the first case.

This shows the following.

Proposition 4.5. The functions $\psi_{e_{i}}, i=1, \ldots, n$ and $\lambda_{\alpha, j}, \alpha=1, \ldots, n-$ $3, j=1,2$, are mutually Poisson commutative.

Proof. This follows from Remark 4.1, Proposition 4.2, Lemma 4.4, and the fact that $\lambda_{\alpha, j}$ and $\psi_{e_{i}}$ are pullbacks of invariant functions.

Since the number $\#\left\{\lambda_{\alpha, j}, \psi_{e_{i}}\right\}=3 n-6$ of functions we have obtained is larger than $(1 / 2) \operatorname{dim}_{\mathbb{R}} \operatorname{Gr}(2, n)=2 n-4$, these functions cannot be functionally independent. In fact, there is one linear relation for each triangle in the triangulation. For example, for a triangle whose edges are three diagonals, say, $d_{1}, d_{2}$, and $d_{3}=d_{1}+d_{2}, U_{1} \times U_{2}$ is a subgroup of $U_{3}$, and the moment map $\mu_{U_{3}}$ has the form

$$
\mu_{U_{3}}=\left(\begin{array}{cc}
\mu_{U_{1}} & * \\
* & \mu_{U_{2}}
\end{array}\right) .
$$

Hence, we have

$$
\begin{aligned}
\lambda_{3,1}+\lambda_{3,2} & =\operatorname{tr} \mu_{U_{3}} \\
& =\operatorname{tr} \mu_{U_{1}}+\operatorname{tr} \mu_{U_{2}} \\
& =\lambda_{1,1}+\lambda_{1,2}+\lambda_{2,1}+\lambda_{2,2} .
\end{aligned}
$$

Set $\psi_{d_{\alpha}}=\lambda_{\alpha, 2}$ for each diagonal $d_{\alpha}$. We claim that

$$
\Psi_{\Gamma}:=\left(\psi_{d_{1}}, \psi_{d_{2}}, \ldots, \psi_{d_{n-3}}, \psi_{e_{1}}, \ldots, \psi_{e_{n-1}}\right): \operatorname{Gr}(2, n) \longrightarrow \mathbb{R}^{2 n-4}
$$

is a completely integrable system on $\operatorname{Gr}(2, n)$. 
Proposition 4.6. We have that $\left(\psi_{d_{1}}, \psi_{d_{2}}, \ldots, \psi_{d_{n-3}}\right)$ induces the bending system $\Phi_{\Gamma}$ on $\mathcal{M}_{r}$ by the symplectic reduction (2.7) up to sign and additive constants.

Proof. Note that the set of nonzero eigenvalues of $A B$ for not necessarily square matrices $A$ and $B$ is bijective with the set of nonzero eigenvalues of $B A$. It follows that the first and second eigenvalues $\lambda_{\alpha, 1}, \lambda_{\alpha, 2}$ of

$$
\mu_{U_{\alpha}}(z, w)=\frac{1}{2}\left(z_{i}, w_{i}\right)_{i \in I_{\alpha}}\left(z_{i}, w_{i}\right)_{i \in I_{\alpha}}^{*} \in \sqrt{-1} \mathfrak{u}\left(\left|I_{\alpha}\right|\right)
$$

coincide with those of

$$
\frac{1}{2}\left(z_{i}, w_{i}\right)_{i \in I_{\alpha}}^{*}\left(z_{i}, w_{i}\right)_{i \in I_{\alpha}}=\frac{1}{2} \sum_{i \in I_{\alpha}}\left(\begin{array}{ll}
\left|z_{i}\right|^{2} & \bar{z}_{i} w_{i} \\
z_{i} \bar{w}_{i} & \left|w_{i}\right|^{2}
\end{array}\right) \in \sqrt{-1} \mathfrak{u}(2) .
$$

Since (4.1) is $T_{U(n)}$-invariant, its eigenvalues $\lambda_{\alpha, j}$ descend to functions on $\mathcal{M}_{r}$. Recall from Section 2 that sides of the polygon are given by

$$
\mu_{\mathrm{SU}(2)}\left(z_{i}, w_{i}\right)=\frac{1}{2}\left(\begin{array}{cc}
\left(\left|z_{i}\right|^{2}-\left|w_{i}\right|^{2}\right) / 2 & \bar{z}_{i} w_{i} \\
z_{i} \bar{w}_{i} & \left(\left|w_{i}\right|^{2}-\left|z_{i}\right|^{2}\right) / 2
\end{array}\right)
$$

considered as an element of $\mathbb{R}^{3}$ by the isomorphism $h: \mathbb{R}^{3} \stackrel{\sim}{\rightarrow} \mathfrak{s u}(2)$. Then (4.1) can be written as

$$
\sum_{i \in I_{\alpha}} \mu_{\mathrm{SU}(2)}\left(z_{i}, w_{i}\right)+\frac{1}{4} \sum_{i \in I_{\alpha}}\left(\begin{array}{cc}
\left|z_{i}\right|^{2}+\left|w_{i}\right|^{2} & 0 \\
0 & \left|z_{i}\right|^{2}+\left|w_{i}\right|^{2}
\end{array}\right)
$$

whose second term is a constant $\sum_{i \in I_{\alpha}} \operatorname{diag}\left(r_{i}, r_{i}\right)$ on the level set $\mu_{T_{U(n)}}^{-1}(2 \boldsymbol{r})$, while the first term $\sum_{i \in I_{\alpha}} \mu_{\mathrm{SU}(2)}\left(z_{i}, w_{i}\right)$, which is the $\alpha$ th diagonal, has eigenvalues $\pm \varphi_{\alpha}$. Hence, we have

$$
\lambda_{\alpha, 2}=-\varphi_{\alpha}+\sum_{i \in I_{\alpha}} r_{i}
$$

on $\mathcal{M}_{r}$.

The next lemma completes the proof of Theorem 1.1.

Lemma 4.7. The functions in $\Psi_{\Gamma}$ are functionally independent. 
Proof. For a function $f$, let $\xi_{f}$ denote its Hamiltonian vector field. From Proposition 4.6 and the fact that the bending Hamiltonians are functionally independent, $\xi_{\psi_{d_{1}}}, \ldots, \xi_{\psi_{d_{n-3}}}$ are linearly independent and transverse to $T_{U(n)}$-orbits on an open dense subset of a level set $\mu_{T_{U(n)}}^{-1}(\boldsymbol{r}) \subset \operatorname{Gr}(2, n)$ for generic $\boldsymbol{r}$. On the other hand, $\xi_{\psi_{e_{1}}}, \ldots, \xi_{\psi_{e_{n-1}}}$ give basis of tangent spaces of $T_{U(n)}$-orbits, and thus $\left\{\xi_{\psi_{d_{\alpha}}}, \xi_{\psi_{e_{i}}}\right\}_{\alpha, i}$ are linearly independent on an open dense subset.

REMARK 4.8. From the facts that the bending Hamiltonians $\varphi_{\alpha}$ are action variables and $\psi_{e_{i}}$ are moment maps of $S^{1}$-actions, the functions $\left(\psi_{d_{\alpha}}, \psi_{e_{i}}\right)_{\alpha, i}$ are also action variables.

Recall that lengths of sides and diagonals of the polygon are given by $r_{i}=\psi_{e_{i}} / 2$ and $\varphi_{d_{\alpha}}=\sum_{i \in I_{\alpha}} \psi_{e_{i}} / 2-\psi_{d_{\alpha}}$. Let $\left(u_{e_{1}}, \ldots, u_{e_{n-1}}, u_{d_{1}}, \ldots, u_{d_{n-3}}\right)$ be the coordinates on $\mathbb{R}^{2 n-4}$ corresponding to $\psi_{e_{i}}$ and $\psi_{d_{\alpha}}$, and define other coordinates corresponding to length functions for $a \in\left\{e_{1}, \ldots, e_{n}, d_{1}, \ldots\right.$, $\left.d_{n-3}\right\}$ by

$$
u(a)= \begin{cases}\frac{1}{2} u_{e_{i}}, & a=e_{i}(i=1, \ldots, n-1), \\ |\boldsymbol{r}|-\frac{1}{2} \sum_{i=1}^{n-1} u_{e_{i}}, & a=e_{n} \\ -u_{d_{\alpha}}+\frac{1}{2} \sum_{i \in I_{\alpha}} u_{e_{i}}, & a=d_{\alpha}\end{cases}
$$

THEOREM 4.9. Let $\Delta_{\Gamma}$ be the polytope in $\mathbb{R}^{2 n-4}$ defined by triangle inequalities

$$
|u(a)-u(b)| \leq u(c) \leq u(a)+u(b)
$$

for each triangle in $\Gamma$ with sides $a, b, c$. Then $\Delta_{\Gamma}$ is the moment polytope of the integrable system: $\Delta_{\Gamma}=\Psi_{\Gamma}(\operatorname{Gr}(2, n))$.

Proof. Note that there exists a polygon with prescribed side and diagonal lengths if they satisfy triangle inequalities. Since the quotient $\operatorname{Gr}(2, n) / T_{U(n)}$ can be regarded as a moduli space of polygons with fixed perimeter, the induced map $\Psi_{\Gamma}: \operatorname{Gr}(2, n) / T_{U(n)} \rightarrow \Delta_{\Gamma}$ is surjective, which proves the theorem.

The image $\Delta_{\Gamma}(\boldsymbol{r})$ of the polygon space $\mathcal{M}_{\boldsymbol{r}}$ is a subset of $\Delta_{\Gamma}$ defined by $\left(u\left(e_{1}\right), \ldots, u\left(e_{n}\right)\right)=\boldsymbol{r}$. Furthermore, we have the following.

Proposition 4.10. Let $\Gamma_{1}$ and $\Gamma_{2}$ be two triangulations which are transformed into each other by a Whitehead move replacing $d$ with $d^{\prime}$. Then 
$\Delta_{\Gamma_{1}}$ is transformed into $\Delta_{\Gamma_{2}}$ by a piecewise-linear transformation (1.3) with respect to $\left(u\left(e_{i}\right), u\left(d_{\alpha}\right)\right)_{i, \alpha}$. This map is defined over $\mathbb{Z}$ with respect to the coordinates $\left(u_{e_{i}}, u_{d_{\alpha}}\right)_{i, \alpha}$ if $|\boldsymbol{r}| \in \mathbb{Z}$. Hence, the volume and the number of integral points in the case where $\Delta_{\Gamma}$ is integral are independent of the choice of $\Gamma$.

Proof. It is obvious that the piecewise-linear map given by (1.3) sends $\Delta_{\Gamma_{1}}$ to $\Delta_{\Gamma_{2}}$, preserving the volumes. We need to show that this map is defined over $\mathbb{Z}$, since the transformation (4.2) is not defined over $\mathbb{Z}$.

Let $a_{1}, \ldots, a_{4} \in\left\{e_{1}, \ldots, e_{n}, d_{1}, \ldots, d_{n-3}\right\}$ be the sides of the quadrilateral having $d$ and $d^{\prime}$ as its diagonals, and take $I_{a_{1}}, \ldots, I_{a_{4}} \subset\{1, \ldots, n\}$ such that $\sum_{i \in I_{a_{k}}} e_{i}= \pm a_{k}$ and $I_{a_{1}} \sqcup \cdots \sqcup I_{a_{4}}=\{1, \ldots, n\}$. We set $I_{d}=I_{a_{1}} \cup I_{a_{2}}$ and $I_{d^{\prime}}=I_{a_{1}} \cup I_{a_{4}}$ so that

$$
\sum_{i \in I_{d}} e_{i}= \pm d, \quad \sum_{i \in I_{d^{\prime}}} e_{i}= \pm d^{\prime} .
$$

Define $v(a)=\sum_{j \in I_{a}} u_{e_{j}} / 2=\sum_{i \in I_{a}} u\left(e_{i}\right)$ for each $a=a_{1}, \ldots, a_{4}, d, d^{\prime}$. Then $u(a) \pm v(a)$ have integral coefficients with respect to $u_{e_{i}}$ and $u_{d_{\alpha}}$. We also note that

$$
v\left(a_{1}\right)+\cdots+v\left(a_{4}\right)=\sum_{i=1}^{n} u\left(e_{i}\right)=|\boldsymbol{r}| .
$$

Then we have

$$
\begin{aligned}
\min & \left(u\left(a_{1}\right)+u\left(a_{2}\right), u\left(a_{3}\right)+u\left(a_{4}\right)\right) \\
= & \min \left(u\left(a_{1}\right)+v\left(a_{1}\right)+u\left(a_{2}\right)+v\left(a_{2}\right), u\left(a_{3}\right)-v\left(a_{3}\right)+u\left(a_{4}\right)-v\left(a_{4}\right)+|\boldsymbol{r}|\right) \\
& -v\left(a_{1}\right)-v\left(a_{2}\right),
\end{aligned}
$$

and the first term in the right-hand side is defined over $\mathbb{Z}$. Similarly, we have

$$
\begin{aligned}
\min & \left(u\left(a_{1}\right)+u\left(a_{4}\right), u\left(a_{2}\right)+u\left(a_{3}\right)\right) \\
= & \min \left(u\left(a_{1}\right)+v\left(a_{1}\right)+u\left(a_{4}\right)+v\left(a_{4}\right), u\left(a_{2}\right)-v\left(a_{2}\right)+u\left(a_{3}\right)-v\left(a_{3}\right)+|\boldsymbol{r}|\right) \\
& -v\left(a_{1}\right)-v\left(a_{4}\right) .
\end{aligned}
$$

Thus, (1.3) can be written as

$$
u\left(d^{\prime}\right)+v\left(a_{1}\right)+v\left(a_{4}\right)=u(d)+v\left(a_{1}\right)+v\left(a_{2}\right)+g(u)
$$

for some $g(u) \in \mathbb{Z}\left[u_{e_{i}}, u_{d_{\alpha}}\right]$. Since $v\left(a_{1}\right)+v\left(a_{2}\right)=v(d)$ and $v\left(a_{1}\right)+v\left(a_{4}\right)=$ $v\left(d^{\prime}\right)$, the above coordinate change is defined over $\mathbb{Z}$. 
EXAmPLe 4.11 (see $[\mathrm{HK}]$ ). Suppose that $\Gamma$ is the caterpillar given by $d_{\alpha}=$ $e_{1}+e_{2}+\cdots+e_{\alpha+1}, \alpha=1, \ldots, n-3$. Then the subgroups $U_{d_{\alpha}} \cong U(\alpha+1)$ satisfy

$$
U_{d_{n-3}} \supset U_{d_{n-4}} \supset \cdots \supset U_{d_{1}} \supset S_{e_{1}}^{1} .
$$

The first and the second eigenvalues of the moment map $\mu_{U_{\alpha}}$ are given by

$$
\lambda_{1}^{(\alpha+1)}:=\lambda_{\alpha, 1}=-\psi_{d_{\alpha}}+\sum_{i=1}^{\alpha+1} \psi_{e_{i}}, \quad \lambda_{2}^{(\alpha+1)}:=\lambda_{\alpha, 2}=\psi_{d_{\alpha}} .
$$

We also consider the action of $U_{-e_{n}} \cong U(n-1)$ corresponding to $I_{-e_{n}}=$ $\{1,2, \ldots, n-1\}$. The eigenvalues of the corresponding moment map $\mu_{U_{-e_{n}}}$ give functions of eigenvalues $\lambda_{1}^{(n-1)} \geq \lambda_{2}^{(n-1)} \geq 0$. Since $\lambda_{1}^{(n-1)}=|\boldsymbol{r}|$ is constant and

$$
\lambda_{2}^{(n-1)}=\sum_{i=1}^{n-1} \psi_{e_{i}}-|\boldsymbol{r}|,
$$

$\Psi_{\Gamma}$ is equivalent to the Gelfand-Cetlin system $\left(\lambda_{j}^{(k)}\right)_{j, k}: \operatorname{Gr}(2, n) \rightarrow \mathbb{R}^{2 n-4}$, where we set $\lambda_{1}^{(1)}=\psi_{e_{1}}$. It is easy to check that the triangle inequalities are equivalent to the inequalities for Gelfand-Cetlin patterns

$|\boldsymbol{r}| \quad \lambda_{2}^{(n-1)}$
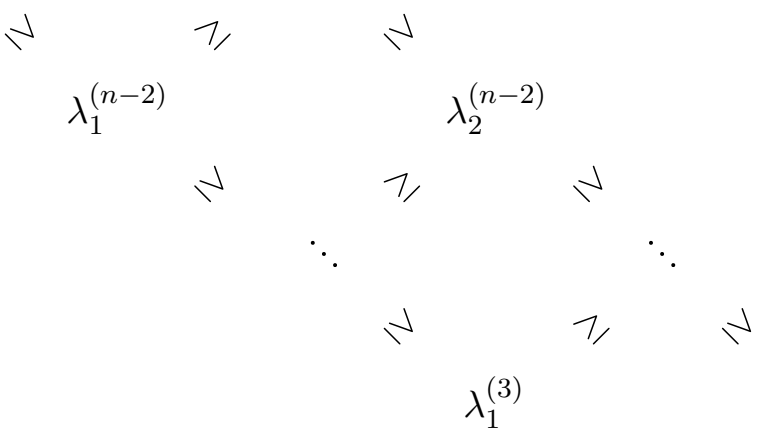

$\lambda_{1}^{(3)}$

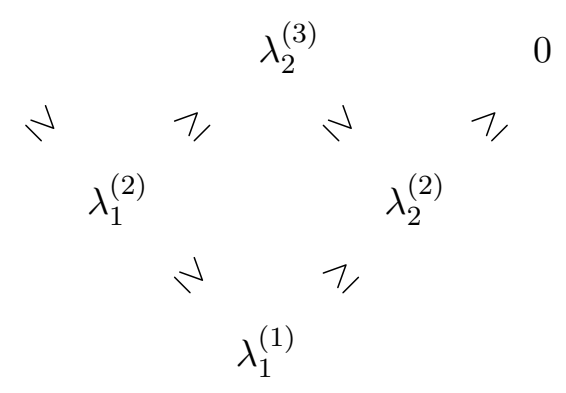




\section{$\S 5$. Degenerations of Grassmannians in stages}

Recall that the Plücker embedding $\operatorname{Gr}(2, n) \hookrightarrow \mathbb{P}\left(\bigwedge^{2} \mathbb{C}^{n}\right)$ is given by

$$
\left[\begin{array}{cc}
z_{1} & w_{1} \\
\vdots & \vdots \\
z_{n} & w_{n}
\end{array}\right] \longmapsto\left[Z_{i j} ; 1 \leq i<j \leq n\right], \quad Z_{i j}=\operatorname{det}\left(\begin{array}{cc}
z_{i} & w_{i} \\
z_{j} & w_{j}
\end{array}\right)
$$

and that the image is defined by the Plücker relations

$$
p_{i j k l}(Z)=Z_{i j} Z_{k l}-Z_{i k} Z_{j l}+Z_{i l} Z_{j k}=0, \quad i<j<k<l .
$$

Toric degenerations of $\operatorname{Gr}(2, n)$ are given by deforming the Plücker relations into binomials. Speyer and Sturmfels [SS] proved that each toric degeneration of $\operatorname{Gr}(2, n)$ corresponds to a triangulation of the reference polygon $P$. In this section, we construct a multiparameter deformation of $\operatorname{Gr}(2, n)$ which is an extension of the 1-parameter family in [SS].

Fix a triangulation of the reference $n$-gon $P$, and let $\Gamma$ be its dual graph. We choose a numbering and orientations of the diagonals $d_{\alpha}=\sum_{i \in I_{\alpha}} e_{i}$ in such a way that either $I_{\alpha} \supset I_{\beta}$ or $I_{\alpha} \cap I_{\beta}=\emptyset$ is satisfied if $\alpha<\beta$. In particular, we assume that $\left|I_{1}\right|=n-2$ and $I_{\alpha} \subset I_{1}$ for all $\alpha \geq 2$. For two leaves $i, j$ of $\Gamma$, let $\gamma(i, j)$ be the unique path in $\Gamma$ connecting $i$ and $j$ (see Figure 9$)$. We introduce a parameter $t_{\alpha}$ for each diagonal $d_{\alpha}$. We define a weight $\boldsymbol{w}_{i j}^{\Gamma}=\left(w_{i j, 1}^{\Gamma}, \ldots, w_{i j, n-3}^{\Gamma}\right)$ of $Z_{i j}$ by

$$
w_{i j, \alpha}^{\Gamma}= \begin{cases}\frac{1}{2} & \text { if } \gamma(i, j) \text { intersects } d_{\alpha}, \\ 0 & \text { otherwise }\end{cases}
$$

and we set

$$
\boldsymbol{t}^{\boldsymbol{w}_{i j}^{\Gamma}}=\prod_{\alpha=1}^{n-3} t_{\alpha}^{w_{i j, \alpha}^{\Gamma}}=\prod_{\alpha} \sqrt{t_{\alpha}}
$$

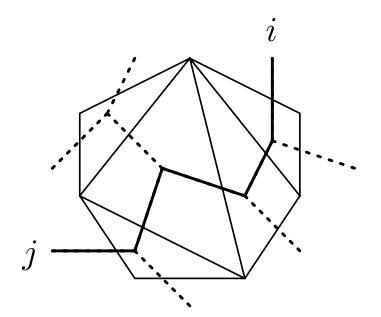

Figure 9: A path $\gamma(i, j)$ connecting the $i$ th and $j$ th leaves. 
where the product on the right-hand side is taken over all $\alpha \in\{1, \ldots, n-3\}$ such that $d_{\alpha}$ crosses $\gamma(i, j)$. For a polynomial $p\left(Z_{i j}\right)$, we define $w_{\alpha}^{\Gamma}(p)$ to be the maximum of weights of monomials in $p$ with respect to $t_{\alpha}$, and we set

$$
\widetilde{p}\left(Z_{i j}, t_{\alpha}\right)=\prod_{\alpha} t_{\alpha}^{w_{\alpha}^{\Gamma}(p)} \cdot p\left(\boldsymbol{t}^{-\boldsymbol{w}_{i j}^{\Gamma}} Z_{i j}\right) .
$$

Then $\widetilde{p}$ is a polynomial in $Z_{i j}$ and $t_{\alpha}$ for each Plücker relation $p=p_{i j k l}$. The degeneration $f_{\Gamma}: \mathfrak{X}_{\Gamma} \rightarrow \mathbb{C}^{n-3}$ of $\operatorname{Gr}(2, n)$ corresponding to $\Gamma$ is given by

$$
\mathfrak{X}_{\Gamma}=\left\{(Z, \boldsymbol{t}) \in \mathbb{P}\left(\bigwedge^{2} \mathbb{C}^{n}\right) \times \mathbb{C}^{n-3} \mid \widetilde{p}_{i j k l}(Z, \boldsymbol{t})=0, i<j<k<l\right\} .
$$

The restriction $\widetilde{p}_{i j k l}(Z, 0)$ to $t_{1}=\cdots=t_{n-3}=0$ is a binomial, and hence the central fiber $X_{\Gamma, 0}=f_{\Gamma}^{-1}(0, \ldots, 0)$ is a toric variety (see [SS, Section 4] or [HMM, Section 4]). We will see in the next section that $\Delta_{\Gamma}=\Psi_{\Gamma}(\operatorname{Gr}(2, n))$ is the moment polytope of $X_{\Gamma, 0}$.

REMARK 5.1. The restriction of $\mathfrak{X}_{\Gamma}$ to the diagonal $t_{1}=\cdots=t_{n-3}=t$ is the family constructed in $[\mathrm{SS}]$ and $[\mathrm{HMM}]$.

EXAMPLE 5.2. Let $n=5$, and consider the triangulation given by $d_{1}=$ $e_{1}+e_{2}$ and $d_{2}=e_{1}+e_{2}+e_{3}$. Then the defining equations of $\mathfrak{X}_{\Gamma}$ are given by

$$
\left\{\begin{array}{l}
t_{1} Z_{12} Z_{34}-Z_{13} Z_{24}+Z_{14} Z_{23}=0 \\
t_{1} Z_{12} Z_{35}-Z_{13} Z_{25}+Z_{15} Z_{23}=0 \\
t_{1} t_{2} Z_{12} Z_{45}-Z_{14} Z_{25}+Z_{15} Z_{24}=0 \\
t_{2} Z_{13} Z_{45}-Z_{14} Z_{35}+Z_{15} Z_{34}=0 \\
t_{2} Z_{23} Z_{45}-Z_{24} Z_{35}+Z_{25} Z_{34}=0
\end{array}\right.
$$

To see the degeneration in more detail, we introduce the following notation. For an $m$-gon $P^{\prime}$ with sides labeled by $1, \ldots, m$, we define

$$
\widetilde{\mathrm{Gr}}_{P^{\prime}}=\mathbb{C}^{m \times 2} / / 0_{0} \mathrm{SU}(2)
$$

to be a cone over the Grassmannian

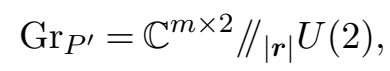

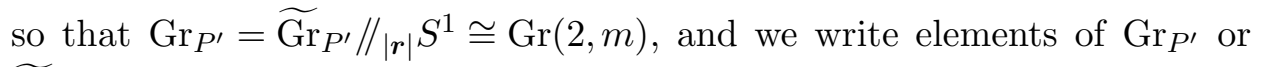
$\widetilde{\mathrm{Gr}}_{P^{\prime}}$ as

$$
\left[\begin{array}{cc}
z_{1}^{P^{\prime}} & w_{1}^{P^{\prime}} \\
\vdots & \vdots \\
z_{m}^{P^{\prime}} & w_{m}^{P^{\prime}}
\end{array}\right]
$$


Let $\widetilde{\operatorname{Gr}}_{P^{\prime}} \hookrightarrow V_{P^{\prime}}:=\bigwedge^{2} \mathbb{C}^{m}$, and let $\operatorname{Gr}_{P^{\prime}} \hookrightarrow \mathbb{P}\left(V_{P^{\prime}}\right)$ be the Plücker embeddings given by

$$
Z_{i j}^{P^{\prime}}=\operatorname{det}\left(\begin{array}{ll}
z_{i}^{P^{\prime}} & w_{i}^{P^{\prime}} \\
z_{j}^{P^{\prime}} & w_{j}^{P^{\prime}}
\end{array}\right) .
$$

Note that if $P^{\prime}$ is a triangle, then $\widetilde{G r}_{P^{\prime}}=V_{P^{\prime}}=\bigwedge^{2} \mathbb{C}^{3} \cong \mathbb{C}^{3}$ and $\operatorname{Gr}_{P^{\prime}} \cong \mathbb{P}^{2}$. For a triangulation $\Gamma^{\prime}$ of $P^{\prime}$, let $\mathfrak{X}_{\Gamma^{\prime}} \rightarrow \mathbb{C}^{m-3}$ and $\widetilde{\mathfrak{X}}_{\Gamma^{\prime}} \rightarrow \mathbb{C}^{m-3}$ denote the corresponding toric degenerations of $\mathrm{Gr}_{P^{\prime}}$ and $\widetilde{\mathrm{Gr}}_{P^{\prime}}$, respectively.

We fix a diagonal $d=d_{\alpha}$ in $\Gamma$, and we consider a 1-parameter subfamily $f_{\Gamma}^{\prime}: \mathfrak{X}_{\Gamma}^{\prime} \rightarrow \mathbb{C}$ of $f_{\Gamma}: \mathfrak{X}_{\Gamma} \rightarrow \mathbb{C}^{n-3}$ defined by $t_{\beta}=1$ for all $\beta \neq \alpha$. Suppose that the diagonal $d$ connects the $p$ th vertex and the $q$ th vertex $(p<q)$, and set

$$
\begin{aligned}
& I_{+}=\{p+1, p+2, \ldots, q\} \\
& I_{-}=\{1, \ldots, p, q+1, \ldots, n\}=\{1, \ldots, n\} \backslash I_{+} .
\end{aligned}
$$

Then $d_{+}=\sum_{i \in I_{+}} e_{i}$ or $d_{-}=\sum_{i \in I_{-}} e_{i}=-d_{+}$coincides with $d$, and $U_{d_{+}} \cap$ $U_{d_{-}}=\{1\}$ in $U(n)$. The defining equations of $\mathfrak{X}_{\Gamma}^{\prime}$ are

$$
\begin{aligned}
& t Z_{i j} Z_{k l}-Z_{i k} Z_{j l}+Z_{i l} Z_{j k}=0, \quad i, j \in I_{ \pm} \text {and } k, l \in I_{\mp}, \\
& Z_{i j} Z_{k l}-Z_{i k} Z_{j l}+t Z_{i l} Z_{j k}=0, \quad i, l \in I_{-} \text {and } j, k \in I_{+}, \\
& Z_{i j} Z_{k l}-Z_{i k} Z_{j l}+Z_{i l} Z_{j k}=0, \quad \text { otherwise. }
\end{aligned}
$$

Then it is easy to see the following.

Lemma 5.3. The family $\mathfrak{X}_{\Gamma}^{\prime} \rightarrow \mathbb{C}$ is $\left(U_{d_{+}} \times U_{d_{-}}\right)$-invariant. In particular, $\mathfrak{X}_{\Gamma}^{\prime}$ admits a natural $U_{d_{\beta}}$-action if $U_{d_{\beta}} \subset U_{d_{+}} \times U_{d_{-}}$.

We study the central fiber of $f_{\Gamma}^{\prime}: \mathfrak{X}_{\Gamma}^{\prime} \rightarrow \mathbb{C}$. Let $P=P_{+} \cup_{d} P_{-}$be the subdivision of the reference polygon by the diagonal $d$, where $P_{ \pm}$is a polygon whose sides are $e_{i}\left(i \in I_{ \pm}\right)$and $d=d_{\alpha}$. Each $P_{ \pm}$has a triangulation $\Gamma_{ \pm}$ induced from $\Gamma$ as in Figure 10. We write elements of $\widetilde{\mathrm{Gr}}_{P_{+}}$as

$$
\left[\begin{array}{cc}
z_{i}^{P_{+}} & w_{i}^{P_{+}} \\
z_{\alpha}^{P_{+}} & w_{\alpha}^{P_{+}}
\end{array}\right]_{i \in I_{+}}=\left[\begin{array}{cc}
z_{p+1}^{P_{+}} & w_{p+1}^{P_{+}} \\
\vdots & \vdots \\
z_{q}^{P_{+}} & w_{q}^{P_{+}} \\
z_{\alpha}^{P_{+}} & w_{\alpha}^{P_{+}}
\end{array}\right]
$$




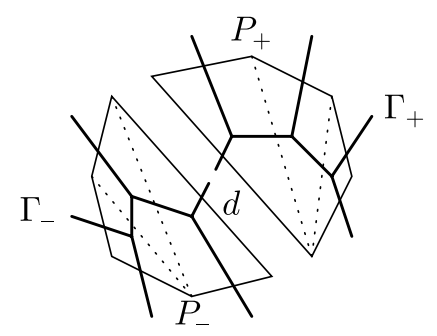

Figure 10: A subdivision of a polygon and the induced trees.

and similarly for $\widetilde{\mathrm{Gr}}_{P_{-}}$. We introduce two groups $S_{0}^{1}$ and $S_{d_{\alpha}}^{1}$ isomorphic to $S^{1}$, and we define an $S_{0}^{1} \times S_{d_{\alpha}}^{1}$-action on $\widetilde{\mathrm{Gr}}_{P_{+}} \times{\widetilde{\mathrm{Gr}_{P_{-}}}}_{\text {by }}$

$$
\begin{gathered}
\left(s_{0}, s_{\alpha}\right) \cdot\left(\left[\begin{array}{cc}
z_{i}^{P_{+}} & w_{i}^{P_{+}} \\
z_{\alpha}^{P_{+}} & w_{\alpha}^{P_{+}}
\end{array}\right]_{i \in I_{+}},\left[\begin{array}{cc}
z_{i}^{P_{-}} & w_{i}^{P_{-}} \\
z_{\alpha}^{P_{-}} & w_{\alpha}^{P_{-}}
\end{array}\right]_{i \in I_{-}}\right) \\
=\left(\left[\begin{array}{cc}
s_{0} z_{i}^{P_{+}} & s_{0} w_{i}^{P_{+}} \\
s_{\alpha} z_{\alpha}^{P_{+}} & s_{\alpha} w_{\alpha}^{P_{+}}
\end{array}\right]_{i \in I_{+}},\left[\begin{array}{cc}
s_{0} z_{i}^{P_{-}} & s_{0} w_{i}^{P_{-}} \\
s_{\alpha}^{-1} z_{\alpha}^{P_{-}} & s_{\alpha}^{-1} w_{\alpha}^{P_{-}}
\end{array}\right]_{i \in I_{-}}\right)
\end{gathered}
$$

for $\left(s_{0}, s_{\alpha}\right) \in S_{0}^{1} \times S_{d_{\alpha}}^{1}$. Let $\mathbb{C}_{0}^{*} \times \mathbb{C}_{d_{\alpha}}^{*}$ be the complexification of $S_{0}^{1} \times S_{d_{\alpha}}^{1}$, which acts on $\widetilde{\mathrm{Gr}}_{P_{+}} \times \widetilde{\mathrm{Gr}}_{P_{-}}$in an obvious way.

Proposition 5.4. The central fiber $X_{\Gamma, 0}^{\prime}=\left(f_{\Gamma}^{\prime}\right)^{-1}(0)$ is isomorphic to the GIT quotient

$$
\widetilde{\mathrm{Gr}}_{P_{+}} \times \widetilde{\mathrm{Gr}}_{P_{-}} / /(2,0) \mathbb{C}_{0}^{*} \times \mathbb{C}_{d_{\alpha}}^{*}
$$

where the polarization is chosen in such a way that the weights of the actions of $\mathbb{C}_{0}^{*} \times \mathbb{C}_{d_{\alpha}}^{*}$ are $(2,0)$. Moreover, the subfamily $\left.\mathfrak{X}_{\Gamma}\right|_{t_{\alpha}=0}$ of $\mathfrak{X}_{\Gamma}$ is induced from the degenerations $\mathfrak{X}_{\Gamma_{ \pm}}$of $\mathrm{Gr}_{P_{ \pm}}$defined by $\Gamma_{ \pm}$:

$$
\left.\mathfrak{X}_{\Gamma}\right|_{t_{\alpha}=0} \cong \widetilde{\mathfrak{X}}_{\Gamma_{+}} \times \widetilde{\mathfrak{X}}_{\Gamma_{-}} / /(2,0) \mathbb{C}_{0}^{*} \times \mathbb{C}_{d_{\alpha}}^{*}
$$

Proof. First note from (5.1) that the defining equations for $X_{\Gamma, 0}^{\prime}$ are

$$
\begin{aligned}
-Z_{i k} Z_{j l}+Z_{i l} Z_{j k} & =0, \quad i, j \in I_{ \pm} \text {and } k, l \in I_{\mp}, \\
Z_{i j} Z_{k l}-Z_{i k} Z_{j l} & =0, \quad i, l \in I_{-} \text {and } j, k \in I_{+}, \\
Z_{i j} Z_{k l}-Z_{i k} Z_{j l}+Z_{i l} Z_{j k} & =0, \quad \text { otherwise. }
\end{aligned}
$$


On the other hand, the $\mathbb{C}_{0}^{*} \times \mathbb{C}_{d_{\alpha}}^{*}$-action on the Plücker coordinates on $V_{P_{ \pm}}$ is given by

$$
Z_{i j}^{P_{ \pm}} \mapsto s_{0}^{2} Z_{i j}^{P_{ \pm}}, \quad Z_{i \alpha}^{P_{ \pm}} \mapsto s_{0} s_{\alpha}^{ \pm 1} Z_{i \alpha}^{P_{ \pm}} \quad\left(i, j \in I_{ \pm}\right)
$$

so that the ring $\mathbb{C}\left[Z_{i j}^{P_{+}}, Z_{i \alpha}^{P_{+}}, Z_{i j}^{P_{-}}, Z_{i \alpha}^{P_{-}}\right]^{\mathbb{C}_{d_{\alpha}}^{*}}$ of $\mathbb{C}_{d_{\alpha}}^{*}$-invariants is generated by $Z_{i j}^{P_{ \pm}}\left(i, j \in I_{ \pm}\right)$and $Z_{i \alpha}^{P_{+}} Z_{j \alpha}^{P_{-}}\left(i \in I_{+}, j \in I_{-}\right)$. Hence, the map $\mathbb{C}\left[Z_{i j}\right] \rightarrow$ $\mathbb{C}\left[Z_{i j}^{P_{+}}, Z_{i \alpha}^{P_{+}}, Z_{i j}^{P_{-}}, Z_{i \alpha}^{P_{-}}\right]$given by

$$
Z_{i j}= \begin{cases}Z_{i j}^{P_{ \pm}}, & i, j \in I_{ \pm}, \\ Z_{i \alpha}^{P_{+}} Z_{j \alpha}^{P_{-}}, & i \in I_{+} \text {and } j \in I_{-}\end{cases}
$$

is a surjection to the invariant ring $\mathbb{C}\left[Z_{i j}^{P_{+}}, Z_{i \alpha}^{P_{+}}, Z_{i j}^{P_{-}}, Z_{i \alpha}^{P_{-}}\right]^{\mathbb{C}_{d_{\alpha}}^{*}}$, and defines an embedding

$$
\widetilde{\mathrm{Gr}}_{P_{+}} \times \widetilde{\mathrm{Gr}}_{P_{-}} / /{ }_{0} \mathbb{C}_{d_{\alpha}}^{*} \hookrightarrow \bigwedge^{2} \mathbb{C}^{n}
$$

of the GIT quotient. It is easy to see that the defining equations for the image of the embedding (5.6) coincide with (5.4), so that $\widetilde{\mathrm{Gr}}_{P_{+}} \times \widetilde{\mathrm{Gr}}_{P_{-}} / /{ }_{0} \mathbb{C}_{d_{\alpha}}^{*}$ is isomorphic to the cone $\tilde{X}_{\Gamma, 0}^{\prime} \subset \bigwedge^{2} \mathbb{C}^{n}$ over $X_{\Gamma, 0}^{\prime}$. Under this identification, the $\mathbb{C}^{*}$-action defining the projection $\widetilde{X}_{\Gamma, 0}^{\prime} \rightarrow X_{\Gamma, 0}^{\prime}$ coincides with the $\mathbb{C}_{0^{-}}^{*}$ action, and hence we have

$$
X_{\Gamma, 0}^{\prime} \cong\left(\widetilde{\mathrm{Gr}}_{P_{+}} \times \widetilde{\mathrm{Gr}}_{P_{-}} / / \mathbb{C}_{d_{\alpha}}^{*}\right) / /{ }_{2} \mathbb{C}_{0}^{*}
$$

It is easy to see that the map (5.5) extends to an isomorphism

$$
\left(\widetilde{\mathfrak{X}}_{\Gamma_{+}} \times \widetilde{\mathfrak{X}}_{\Gamma_{-}} / /{ }_{0} \mathbb{C}_{d_{\alpha}}^{*}\right) / /\left.{ }_{2} \mathbb{C}_{0}^{*} \longrightarrow \mathfrak{X}_{\Gamma}\right|_{t_{\alpha=0}},
$$

and Proposition 5.4 is proved.

REMARK 5.5. From the proof, there is a $\mathbb{C}_{0}^{*}$-action on $\widetilde{X}_{\Gamma, 0}^{\prime} \cong \widetilde{\mathrm{Gr}}_{P_{+}} \times$ $\widetilde{\mathrm{Gr}}_{P_{-}} / /{ }_{0} \mathbb{C}_{d_{\alpha}}^{*}$ such that the coordinates $Z_{i j}$ have weights $1 ; \widetilde{X}_{\Gamma, 0}^{\prime} / /{ }_{2} \mathbb{C}_{0}^{*}$ and $\tilde{X}_{\Gamma, 0}^{\prime} / /{ }_{1} \mathbb{C}_{0}^{*}$ are isomorphic as algebraic varieties (without polarizations). We will write $\left(\widetilde{\mathrm{Gr}}_{P_{+}} \times \widetilde{\mathrm{Gr}}_{P_{-}} / /{ }_{0} \mathbb{C}_{d_{\alpha}}^{*}\right) / /{ }_{1} \mathbb{C}_{0}^{*}$ as $\widetilde{\mathrm{Gr}}_{P_{+}} \times \widetilde{\mathrm{Gr}}_{P_{-}} / /(1,0) \mathbb{C}_{0}^{*} \times \mathbb{C}_{d_{\alpha}}^{*}$ for simplicity.

We now define a degeneration of $\operatorname{Gr}(2, n)$ in stages as follows. For $\alpha=$ $1, \ldots, n-3$, let $f_{\Gamma}^{(\alpha)}: \mathfrak{X}_{\Gamma}^{(\alpha)} \rightarrow \mathbb{C}$ be a subfamily of $\mathfrak{X}_{\Gamma}$ defined by

$$
t_{1}=\cdots=t_{\alpha-1}=0, \quad t_{\alpha+1}=\cdots=t_{n-3}=1,
$$


and write its fibers as $X_{\Gamma, t}^{(\alpha)}=\left(f_{\Gamma}^{(\alpha)}\right)^{-1}(t)$. Then $\mathfrak{X}_{\Gamma}^{(\alpha)}(\alpha=1, \ldots, n-3)$ gives a sequence of families such that $X_{\Gamma, 1}^{(1)}=\operatorname{Gr}(2, n), X_{\Gamma, 0}^{(n-3)}=X_{\Gamma, 0}$, and $X_{\Gamma, 0}^{(\alpha)}=$ $X_{\Gamma, 1}^{(\alpha+1)}$ for each $\alpha$. Furthermore, from the choice of the numbering of the diagonals, $\mathfrak{X}_{\Gamma}^{(\alpha)}$ admits actions of $U_{d_{\beta}}$ for all $\beta \geq \alpha$. Let

$$
P=P_{1}^{(\alpha)} \cup \cdots \cup P_{\alpha+1}^{(\alpha)}
$$

be the subdivision of $P$ given by the diagonals $d_{1}, \ldots, d_{\alpha}$, and let $\Gamma_{m}^{(\alpha)}$ be the triangulation of $P_{m}^{(\alpha)}$ induced from $\Gamma$. For each diagonal $d_{\beta}$, we introduce $S_{d_{\beta}}^{1} \cong S^{1}$ and its complexification $\mathbb{C}_{d_{\beta}}^{*}$, and we extend (5.2) to a $\mathbb{C}_{0}^{*} \times \mathbb{C}_{d_{1}}^{*} \times$ $\cdots \times \mathbb{C}_{d_{\alpha}}^{*}$-action on $\widetilde{\mathrm{Gr}}_{P_{1}^{(\alpha)}} \times \cdots \times \widetilde{\mathrm{Gr}}_{P_{\alpha+1}^{(\alpha)}}$ in an obvious manner. Namely, $\mathbb{C}_{0}^{*}$ acts diagonally on the coordinates having a side $e_{1}, \ldots, e_{n}$ of $P$ in its index, while $\mathbb{C}_{d_{\beta}}^{*}$ acts antidiagonally on the coordinates indexed the diagonal $d_{\beta}$. Then Proposition 5.4 implies the following.

Corollary 5.6. The central fiber $X_{\Gamma, 0}^{(\alpha)} \subset \mathbb{P}\left(\bigwedge^{2} \mathbb{C}^{n}\right)$ of the ath stage $\mathfrak{X}_{\Gamma}^{(\alpha)}$ is isomorphic to

$$
\widetilde{\mathrm{Gr}}_{P_{1}^{(\alpha)}} \times \cdots \times \widetilde{\mathrm{Gr}}_{P_{\alpha+1}^{(\alpha)}} / /(1,0, \ldots, 0) \mathbb{C}_{0}^{*} \times \mathbb{C}_{d_{1}}^{*} \times \cdots \times \mathbb{C}_{d_{\alpha}}^{*}
$$

where the weight of the $\mathbb{C}_{0}^{*} \times \mathbb{C}_{d_{1}}^{*} \times \cdots \times \mathbb{C}_{d_{\alpha}}^{*}$-action is $(1,0, \ldots, 0)$. Moreover, the $(\alpha+1)$ st stage of the degeneration is given by

$$
\mathfrak{X}_{\Gamma}^{(\alpha+1)} \cong \widetilde{\mathfrak{X}}_{\Gamma_{1}^{(\alpha)}}^{(1)} \times \widetilde{\mathrm{Gr}}_{P_{2}^{(\alpha)}} \times \cdots \times \widetilde{\mathrm{Gr}}_{P_{\alpha+1}^{(\alpha)}} / /(1,0, \ldots, 0) \mathbb{C}_{0}^{*} \times \mathbb{C}_{d_{1}}^{*} \times \cdots \times \mathbb{C}_{d_{\alpha}}^{*},
$$

where we assume that $d_{\alpha+1}$ is a diagonal of $P_{1}^{(\alpha)}$, and $\mathfrak{X}_{\Gamma_{1}^{(\alpha)}}^{(1)}$ is the first stage of the degeneration of $\mathrm{Gr}_{P_{1}^{(\alpha)}}$ corresponding to $d_{\alpha+1}$.

Now let us look at the last stage $\alpha=n-3$ of the degeneration. The reference polygon is divided as

$$
P=P_{1}^{(n-3)} \cup \cdots \cup P_{n-2}^{(n-3)},
$$

where all $P_{m}^{(n-3)}(1 \leq m \leq n-2)$ are triangles, and we have

$$
\widetilde{\mathrm{Gr}}_{P_{m}^{(n-3)}}=V_{P_{m}^{(n-3)}}=\operatorname{Spec} \mathbb{C}\left[Z_{a b}^{P_{m}^{(n-3)}}\right] \cong \bigwedge^{2} \mathbb{C}^{3}
$$

where $a, b \in\left\{e_{1}, \ldots, e_{n}, d_{1}, \ldots, d_{n-3}\right\}$ are edges in the triangle $P_{m}^{(n-3)}$. 
Corollary 5.7 ([HMM, Theorem 1.8]). The central fiber $X_{\Gamma}:=X_{\Gamma, 0}$ of $\mathfrak{X}_{\Gamma}$ is a toric variety given by

$$
\begin{aligned}
& V_{P_{1}^{(n-3)}} \times \cdots \times V_{P_{n-2}^{(n-3)}} / /(1,0, \ldots, 0) \\
& \quad \cong\left(\bigwedge^{2} \mathbb{C}^{3}\right)^{n-2} / /\left(\mathbb{C}^{*}\right)^{n-2}
\end{aligned}
$$

The cone

$$
\begin{aligned}
& V_{P_{1}^{(n-3)}} \times \cdots \times V_{P_{n-2}^{(n-3)}} / /(0, \ldots, 0) \\
& \quad=\operatorname{Spec}\left(\mathbb{C}\left[Z_{a b}^{P_{d_{1}}^{(n-3)}}\right]^{\left.\mathbb{C}_{d_{1}}^{*} \times \cdots \times \mathbb{C}_{d_{n-3}}^{*}\right)}\right.
\end{aligned}
$$

over $X_{\Gamma}$ is embedded into the affine space $\bigwedge^{2} \mathbb{C}^{n}$ by the surjection

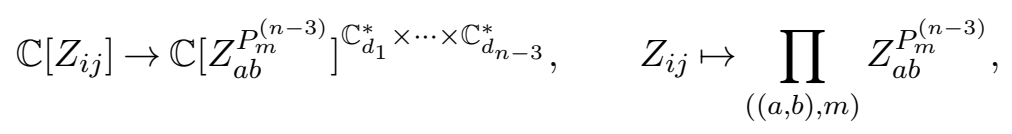

where the product on the right-hand side runs over triples $((a, b), m)$ of edges $(a, b)$ of a triangle $P_{m}^{(n-3)}$ in the triangulation $P=P_{1}^{(n-3)} \cup \cdots \cup P_{n-2}^{(n-3)}$ crossed by the path $\gamma(i, j)$ connecting the $i$ th and the $j$ th leaves as in Figure 9.

Defining equations for the image of the embedding $X_{\Gamma} \hookrightarrow \mathbb{P}\left(\bigwedge^{2} \mathbb{C}^{n}\right)$ come from those of the embedding

$$
V_{P_{a}} \times V_{P_{b}} /{ }_{0} \mathbb{C}_{d_{\alpha}}^{*} \hookrightarrow \bigwedge^{2} \mathbb{C}^{4}
$$

where $P_{a}=P_{a}^{(n-3)}$ and $P_{b}=P_{b}^{(n-3)}$ are two triangles sharing a diagonal $d=d_{\alpha}$. Let $a_{1}$ and $a_{2}$ (resp., $b_{1}$ and $b_{2}$ ) be the remaining edges of $P_{a}$ (resp., $\left.P_{b}\right)$. Let $P_{c}=P_{c}^{(n-2)}=P_{a} \cup P_{b}$ be a quadrilateral obtained as the union of $P_{a}$ and $P_{b}$. Then the inclusion is defined by the homomorphism

$$
\begin{aligned}
& Z_{a_{1} b_{1}}^{P_{c}}=Z_{a_{1} d}^{P_{a}} Z_{b_{1} d}^{P_{b}}, \\
& Z_{a_{1} b_{2}}^{P_{c}}=Z_{a_{1} d}^{P_{a}} Z_{b_{2} d}^{P_{b}}, \\
& Z_{a_{2} b_{1}}^{P_{c}}=Z_{a_{2} d}^{P_{a}} Z_{b_{1} d}^{P_{b}}, \\
& Z_{a_{2} b_{2}}^{P_{c}}=Z_{a_{2} d}^{P_{a}} Z_{b_{2} d}^{P_{b}}, \\
& Z_{a_{1} a_{2}}^{P_{c}}=Z_{a_{1} a_{2}}^{P_{a}},
\end{aligned}
$$




$$
Z_{b_{1} b_{2}}^{P_{c}}=Z_{b_{1} b_{2}}^{P_{b}}
$$

so that the defining equation of the image is given by

$$
Z_{a_{1} b_{1}}^{P_{c}} Z_{a_{2} b_{2}}^{P_{c}}=Z_{a_{1} b_{2}}^{P_{c}} Z_{a_{2} b_{1}}^{P_{c}}
$$

It follows that the singular locus of $V_{P_{a}} \times V_{P_{b}} / /{ }_{0} \mathbb{C}_{d_{\alpha}}^{*}$ is given by

$$
Z_{a_{1} b_{1}}^{P_{c}}=Z_{a_{1} b_{2}}^{P_{c}}=Z_{a_{2} b_{1}}^{P_{c}}=Z_{a_{2} b_{2}}^{P_{c}}=0
$$

that is,

$$
Z_{a_{1} d}^{P_{a}}=Z_{a_{2} d}^{P_{a}}=0 \quad \text { or } \quad Z_{d b_{1}}^{P_{b}}=Z_{d b_{2}}^{P_{b}}=0,
$$

with $Z_{a_{1} a_{2}}^{P_{a}}$ and $Z_{b_{1} b_{2}}^{P_{b}}$ arbitrary. This gives a codimension 3 singularity in $X_{\Gamma}$. Since $X_{\Gamma}$ is a toric variety obtained as the quotient of an affine space by torus action, a singular point of $X_{\Gamma}$ comes from a point on the affine space $V_{P_{1}^{(n-3)}} \times \cdots \times V_{P_{n-2}^{(n-3)}}$ where the torus action has a nontrivial stabilizer. Such a point is contained in one of the loci defined by (5.7), and one obtains the following.

Proposition 5.8. The singular locus $\operatorname{Sing}\left(X_{\Gamma}\right)$ of $X_{\Gamma}$ is the union

$$
\operatorname{Sing}\left(X_{\Gamma}\right)_{A}=\bigcup_{\alpha=1}^{n-3} \operatorname{Sing}\left(X_{\Gamma}\right)_{\alpha}
$$

where

$$
\operatorname{Sing}\left(X_{\Gamma}\right)_{\alpha}=\left\{Z_{a_{1} d_{\alpha}}^{P_{a}}=Z_{a_{2} d_{\alpha}}^{P_{a}}=0 \text { or } Z_{b_{1} d_{\alpha}}^{P_{b}}=Z_{b_{2} d_{\alpha}}^{P_{b}}=0\right\}
$$

\section{$\S 6$. Toric degeneration of the integrable system}

The following definition is introduced in [NNU1, Definition 1.1].

Definition 6.1. Let $\Phi: X \rightarrow \mathbb{R}^{N}$ be a completely integrable system on a projective Kähler manifold $(X, \omega)$. A toric degeneration of $\Phi$ consists of a flat family $f: \mathfrak{X} \rightarrow B$ of algebraic varieties over a complex manifold $B$, a Kähler form $\widetilde{\omega}$ on the smooth locus of $\mathfrak{X}$, a piecewise-smooth path $\gamma:[0,1] \rightarrow$ $B$, a continuous map $\widetilde{\Phi}:\left.\mathfrak{X}\right|_{\gamma([0,1])} \rightarrow \mathbb{R}^{N}$ on $\left.\mathfrak{X}\right|_{\gamma([0,1])}=f^{-1}(\gamma([0,1]))$, and a flow $\phi_{t}$ on $\left.\mathfrak{X}\right|_{\gamma([0,1])}$ which covers the path $\gamma$ and is defined away from the union $\bigcup_{t \in[0,1]} \operatorname{Sing}\left(X_{t}\right)$ of the singular loci of the fibers $X_{t}=f^{-1}(\gamma(t))$ such that 
- for each $t \in[0,1]$, the restriction $\Phi_{t}=\left.\widetilde{\Phi}\right|_{X_{t}}$ is a completely integrable system on the Kähler variety $\left(X_{t}, \omega_{t}=\left.\widetilde{\omega}\right|_{X_{t}}\right)$, whose image $\Phi_{t}\left(X_{t}\right)$ is a convex polytope $\Delta$ independent of $t$;

- $\left(X_{1}, \omega_{1}\right)$ is isomorphic to $(X, \omega)$ as a Kähler manifold;

- $\Phi_{1}$ coincides with $\Phi$ under the above isomorphism $X_{1} \cong X$;

- $\left(X_{0}, \omega_{0}\right)$ is a toric variety with a torus-invariant Kähler form;

- $\Phi_{0}: X_{0} \rightarrow \mathbb{R}^{N}$ is the moment map for the torus action on $X_{0}$ (hence, $\Delta$ is a moment polytope of $X_{0}$ ); and

- for each $t$, there is an open dense subset $X_{t}^{\circ} \subset X_{t}$ such that the flow $\phi_{t}$ sends $X_{t^{\prime}}^{\circ}$ to another fiber $X_{t^{\prime}-t}^{\circ}$, preserving the symplectic structures and the completely integrable systems:

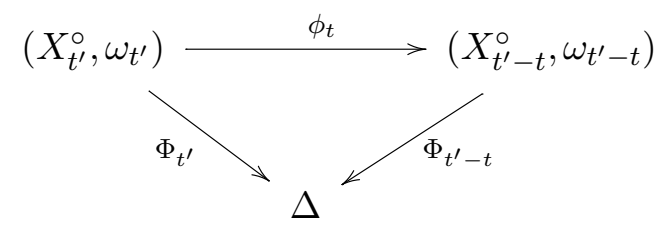

In this section we construct a toric degeneration of $\Psi_{\Gamma}: \operatorname{Gr}(2, n) \rightarrow \mathbb{R}^{2 n-4}$. We consider the family $f_{\Gamma}: \mathfrak{X}_{\Gamma} \rightarrow \mathbb{C}^{n-3}$ constructed in Section 5 , and we let $\gamma$ be a piecewise-linear path connecting the vertices

$$
(1, \ldots, 1),(0,1, \ldots, 1), \ldots,(0, \ldots, 0,1),(0, \ldots, 0) .
$$

Then the restriction of $\mathfrak{X}_{\Gamma}$ to $\gamma$ is the degeneration in stages. We take a Kähler form $\widetilde{\omega}_{r}$ on $\mathfrak{X}_{\Gamma}$ such that the restriction $\omega_{r, t}=\left.\widetilde{\omega}_{\boldsymbol{r}}\right|_{X_{\Gamma, t}}$ to each fiber of $f_{\Gamma}$ is the constant multiple $\left.|\boldsymbol{r}| \cdot \omega_{\mathrm{FS}}\right|_{X_{\Gamma, t}}$ of the Fubini-Study form $\omega_{\mathrm{FS}}$ on $\mathbb{P}\left(\bigwedge^{2} \mathbb{C}^{n}\right)$. For each stage $f_{\Gamma}^{(\alpha)}: \mathfrak{X}_{\Gamma}^{(\alpha)} \rightarrow \mathbb{C}$ of the degeneration, we define a map

$$
\widetilde{\Psi}_{\Gamma}^{(\alpha)}: \mathfrak{X}_{\Gamma}^{(\alpha)} \longrightarrow \mathbb{R}^{2 n-4}
$$

as follows. Recall that $P_{1}^{(\alpha-1)}, \ldots, P_{\alpha}^{(\alpha-1)}$ are subpolygons obtained by cutting $P$ along the diagonals $d_{1}, \ldots, d_{\alpha-1}$ and that $\Gamma_{m}^{(\alpha-1)}$ is the triangulation of $P_{m}^{(\alpha-1)}$ induced from $\Gamma$. From Corollary 5.6, we have

$$
\mathfrak{X}_{\Gamma}^{(\alpha)} \cong \widetilde{\mathfrak{X}}_{\Gamma_{1}^{(\alpha-1)}}^{(1)} \times \widetilde{\mathrm{Gr}}_{P_{2}^{(\alpha-1)}} \times \cdots \times \widetilde{\mathrm{Gr}}_{P_{\alpha}^{(\alpha-1)}} / / \mathbb{C}_{0}^{*} \times \mathbb{C}_{d_{1}}^{*} \times \cdots \times \mathbb{C}_{d_{\alpha-1}}^{*},
$$

where we assume that $d_{\alpha}$ is a diagonal in $P_{1}^{(\alpha-1)}$. The actions of $U_{d_{\beta}}$ for $\beta \geq \alpha$ and $S_{e_{i}}^{1}$ are induced from those on $\widetilde{\mathfrak{X}}_{\Gamma_{1}^{(\alpha-1)}}^{(1)}$ or $\widetilde{\mathrm{Gr}}_{P_{m}^{(\alpha-1)}}$ for some $m$. 
We define

$$
\widetilde{\lambda}_{\beta, j}: \mathfrak{X}_{\Gamma}^{(\alpha)} \longrightarrow \mathbb{R}, \quad j=1,2
$$

to be the first and second eigenvalues of the values of the moment map of the $U_{d_{\beta}}$-action, which is a natural extension of $\lambda_{\beta, j}$. We also extend the moment map $\psi_{e_{i}}$ of the $S_{i}^{1}$-action to

$$
\widetilde{\psi}_{e_{i}}: \mathfrak{X}_{\Gamma}^{(\alpha)} \longrightarrow \mathbb{R}
$$

for $i=1, \ldots, n$. The space $\mathfrak{X}_{\Gamma}^{(\alpha)}$ has an action of another torus $S_{d_{1}}^{1} \times \cdots \times S_{d_{\alpha}}^{1}$, where $S_{d_{\gamma}}^{1}(1 \leq \gamma \leq \alpha)$ acts diagonally on Plücker coordinates $Z_{a d_{\gamma}}^{P_{m}^{(\alpha-1)}}$ having $d_{\gamma}$ in their indices. In other words, the weight of a (Plücker) coordinate $Z_{i j}$ of $\mathbb{P}\left(\bigwedge^{2} \mathbb{C}^{n}\right)$ with respect to the $S_{d_{\gamma}}^{1}$-action is 1 if the path $\gamma(i, j)$ from $i$ to $j$ crosses $d_{\gamma}$, and 0 otherwise. Let

$$
\widetilde{\mu}_{S_{\gamma}^{1}}: \mathfrak{X}_{\Gamma}^{(\alpha)} \longrightarrow \mathbb{R}
$$

be the moment map of the $S_{d_{\gamma}}^{1}$-action, and set $\widetilde{\nu}_{\gamma}=|\boldsymbol{r}|-\widetilde{\mu}_{S_{\gamma}^{1}}$. We define $\widetilde{\Psi}_{\Gamma}^{(\alpha)}: \mathfrak{X}_{\Gamma}^{(\alpha)} \rightarrow \mathbb{R}^{2 n-4}$ by

$$
\widetilde{\Psi}_{\Gamma}^{(\alpha)}=\left(\widetilde{\nu}_{1}, \ldots, \widetilde{\nu}_{\alpha-1}, \widetilde{\lambda}_{\alpha, 2}, \ldots, \widetilde{\lambda}_{n-3,2}, \widetilde{\psi}_{e_{1}}, \ldots, \widetilde{\psi}_{e_{n-1}}\right),
$$

and $\Psi_{\Gamma, t}^{(\alpha)}=\left.\widetilde{\Psi}_{\Gamma}^{(\alpha)}\right|_{X_{\Gamma, t}^{(\alpha)}}$. Then $\Psi_{\Gamma, 1}^{(1)}=\Psi_{\Gamma}$ on $X_{\Gamma, 1}^{(1)}=\operatorname{Gr}(2, n)$ from the construction.

We define $\phi_{t}^{(\alpha)}$ on $\mathfrak{X}_{\Gamma}^{(\alpha)}$ to be the gradient-Hamiltonian flow of $f_{\Gamma}^{(\alpha)}$ introduced by Ruan [R1]. Regarding $f_{\Gamma}^{(\alpha)}: \mathfrak{X}_{\Gamma}^{(\alpha)} \rightarrow \mathbb{C}$ as a holomorphic function, the normalized gradient-Hamiltonian vector field is defined by

$$
\xi^{(\alpha)}:=-\frac{\nabla\left(\Re f_{\Gamma}^{(\alpha)}\right)}{\left|\nabla\left(\Re f_{\Gamma}^{(\alpha)}\right)\right|^{2}}=\frac{\xi_{\Im f_{\Gamma}^{(\alpha)}}}{\left|\xi_{\Im f_{\Gamma}^{(\alpha)}}\right|^{2}},
$$

where $\nabla\left(\Re f_{\Gamma}^{(\alpha)}\right)$ is the gradient vector field of the real part of $f_{\Gamma}^{(\alpha)}$, and $\xi_{\Im f_{\Gamma}^{(\alpha)}}$ is the Hamiltonian vector field of the imaginary part of $f_{\Gamma}^{(\alpha)}$. It is shown in [R1] that $\xi^{(\alpha)}$ is defined on the smooth locus of fibers $X_{\Gamma, t}^{(\alpha)}$, and its flow $\phi_{t}^{(\alpha)}=\exp t \xi^{(\alpha)}$ gives a symplectomorphism

$$
\phi_{1-t}^{(\alpha)}:\left(W_{\Gamma, 1}^{(\alpha)}, \omega_{r, 1}\right) \longrightarrow\left(W_{\Gamma, t}^{(\alpha)}, \omega_{r, t}\right)
$$

for some open subsets $W_{\Gamma, t}^{(\alpha)} \subset X_{\Gamma, t}^{(\alpha)}$. 
REMARK 6.2. The authors do not know whether $\phi_{1-t}^{(\alpha)}$ can be extended to $X_{\Gamma, 1}^{(\alpha)} \rightarrow X_{\Gamma, t}^{(\alpha)}$. Note that the total space of the family is not smooth in general, and hence we cannot apply the analysis in [R2].

We begin with the proof of Theorem 1.2 for the first stage.

LEMma 6.3. We have that $\Psi_{\Gamma, t}^{(1)}: X_{\Gamma, t}^{(1)} \rightarrow \mathbb{R}^{2 n-4}$ is a completely integrable system for each $t$. The gradient-Hamiltonian flow $\phi_{t}^{(1)}$ is defined on an open dense subset $W_{\Gamma, t}^{(1)} \subset X_{\Gamma, t}^{(1)}$ and gives a symplectomorphism preserving the completely integrable systems; that is,

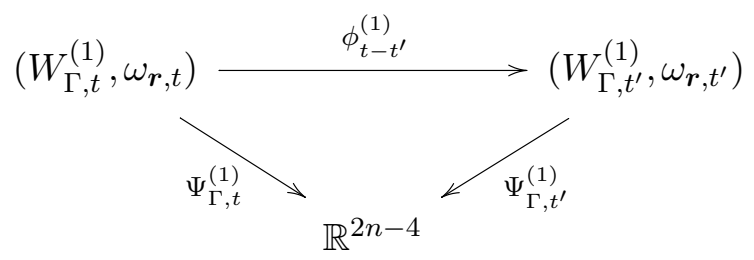

commutes. Furthermore, $\Psi_{\Gamma, 0}^{(1)}$ coincides with $\Psi_{\Gamma, 1}^{(2)}$ on $X_{\Gamma, 0}^{(1)}=X_{\Gamma, 1}^{(2)}$.

Proof. Poisson commutativity follows from the same argument as in the proof of Proposition 4.5. The fact that $f_{\Gamma}^{(1)}$ is invariant under the actions of $U_{d_{\beta}}$ and $S_{e_{i}}^{1}$ implies that $\phi_{t}^{(1)}$ preserves the completely integrable system just as in [NNU1, Section 7]. Since $X_{\Gamma, t}^{(1)}$ is smooth for $t \neq 0, \phi_{1-t}^{(1)}$ is defined on $X_{\Gamma, 1}^{(1)}$, and

$$
\phi_{1-t}^{(1)}: X_{\Gamma, 1}^{(1)} \longrightarrow X_{\Gamma, t}^{(1)}
$$

is a symplectomorphism satisfying $\Psi_{\Gamma, 1}^{(1)}=\phi_{1-t}^{(1)}{ }^{*} \Psi_{\Gamma, t}^{(1)}$. Hence, the functions in $\Psi_{\Gamma, t}^{(1)}$ are functionally independent on an open dense subset of $X_{\Gamma, t}^{(1)}$.

For $t=0$, there is an open subset $W_{\Gamma, 1}^{(1)} \subset X_{\Gamma, 1}^{(1)}$ on which $\phi_{1}^{(1)}$ is defined and gives a symplectomorphism

$$
\phi_{1}^{(1)}: W_{\Gamma, 1}^{(1)} \longrightarrow X_{\Gamma, 0}^{(1)} \backslash \operatorname{Sing}\left(X_{\Gamma, 0}^{(1)}\right) .
$$

From $\Psi_{\Gamma, 1}^{(1)}=\phi_{1}^{(1)^{*}} \Psi_{\Gamma, 0}^{(1)}$ and the fact that $\Psi_{\Gamma, 1}^{(1)}$ is functionally independent, $\Psi_{\Gamma, 0}^{(1)}$ is also functionally independent. For each $t$, set $W_{\Gamma, t}^{(1)}=\phi_{1-t}^{(1)}\left(W_{\Gamma, 1}^{(1)}\right)$. To show that $W_{\Gamma, t}^{(1)}$ is dense in $X_{\Gamma, t}^{(1)}$, we consider the inverse image $\operatorname{Int}\left(X_{\Gamma, t}^{(1)}\right)=$ 
$\left(\Psi_{\Gamma, t}^{(1)}\right)^{-1}\left(\operatorname{Int}\left(\Delta_{\Gamma}\right)\right)$ of the interior of $\Delta_{\Gamma}$. Note that

$$
\operatorname{Int}\left(X_{\Gamma, t}^{(1)}\right) \cong T^{2 n-4} \times \operatorname{Int}\left(\Delta_{\Gamma}\right)
$$

is a dense subset in $X_{\Gamma, t}^{(1)}$ on which $\Psi_{\Gamma, t}^{(1)}$ defines a free $T^{2 n-4}$-action. We also note that $\operatorname{Int}\left(X_{\Gamma, 0}^{(1)}\right)$ is contained in the smooth locus of $X_{\Gamma, 0}^{(1)}$. Since $\phi_{t}^{(1)}$ preserves the Hamiltonian torus actions, we have a symplectomorphism $\phi_{t}^{(1)}: \operatorname{Int}\left(X_{\Gamma, t}^{(1)}\right) \rightarrow \operatorname{Int}\left(X_{\Gamma, 0}^{(1)}\right)$. Hence, we have $\operatorname{Int}\left(X_{\Gamma, t}^{(1)}\right) \subset W_{\Gamma, t}^{(1)}$, which shows that $W_{\Gamma, t}^{(1)}$ is dense in $X_{\Gamma, t}^{(1)}$.

Finally, we show that $\widetilde{\lambda}_{1,2}$ coincides with $\widetilde{\nu}_{1}$ on $X_{\Gamma, 0}^{(1)}=X_{\Gamma, 1}^{(2)}$. Let $P=$ $P_{1}^{(1)} \cup_{d_{1}} P_{2}^{(1)}$ be the subdivision of $P$ given by $d_{1}=\sum_{i \in I_{1}} e_{i}$ such that $P_{2}^{(1)}$ is a triangle. Then $X_{\Gamma, 0}^{(1)}$ is written as

$$
X_{\Gamma, 0}^{(1)} \cong \widetilde{\mathrm{Gr}}_{P_{1}^{(1)}} \times V_{P_{2}^{(1)} / /(1,0)} \mathbb{C}_{0}^{*} \times \mathbb{C}_{d_{1}}^{*}
$$

From the construction, $\widetilde{\lambda}_{1,2}, \ldots, \widetilde{\lambda}_{n-3,2}$ and $\widetilde{\psi}_{e_{i}}\left(i \in I_{1}\right)$ are induced from the completely integrable system $\Psi_{\Gamma_{1}^{(1)}}$ on $\operatorname{Gr}_{P_{1}^{(1)}}$, while $\widetilde{\psi}_{e_{i}}\left(i \notin I_{1}\right)$ are induced from a natural $T^{2}$-action on $V_{P_{2}^{(1)}} \cong \mathbb{C}^{3}$. Note that $U_{d_{1}} \times S_{d_{1}}^{1} \cong U(n-2) \times$ $U(1)$ is a subgroup of $U(n-1)$ which naturally acts on $\operatorname{Gr}_{P_{1}^{(1)}}=\operatorname{Gr}(2, n-$ 1). Since $\mathrm{Gr}_{P_{1}^{(1)}}$ is symplectically identified with the (co)adjoint orbit of $\operatorname{diag}(|\boldsymbol{r}|,|\boldsymbol{r}|, 0, \ldots, 0)$, values of the moment map $\mu_{U(n-1)}$ on $\mathrm{Gr}_{P_{1}^{(1)}}$ have constant eigenvalues $|\boldsymbol{r}|,|\boldsymbol{r}|, 0, \ldots, 0$. Then the eigenvalues of $\mu_{U_{d_{1}}}$ satisfy

$$
|\boldsymbol{r}| \geq \widetilde{\lambda}_{1,1} \geq|\boldsymbol{r}| \geq \widetilde{\lambda}_{1,2} \geq 0
$$

Thus, we have

$$
2|\boldsymbol{r}|=\operatorname{tr} \mu_{U(n-1)}=\operatorname{tr} \mu_{U_{d_{1}}}+\widetilde{\mu}_{S_{d_{1}}^{1}}=|\boldsymbol{r}|+\widetilde{\lambda}_{1,2}+\widetilde{\mu}_{S_{d_{1}}^{1}}
$$

or, equivalently,

$$
\widetilde{\mu}_{S_{d_{1}}^{1}}=-\widetilde{\lambda}_{1,2}+|\boldsymbol{r}|
$$

as desired.

The next proposition completes the proof of Theorem 1.2. 
Proposition 6.4. For each $t, \Psi_{\Gamma, t}^{(\alpha)}: X_{\Gamma, t}^{(\alpha)} \rightarrow \mathbb{R}^{2 n-4}$ is a completely integrable system. The gradient-Hamiltonian flow $\phi_{t}^{(\alpha)}$ is defined on an open dense subset $W_{\Gamma, t}^{(\alpha)} \subset X_{\Gamma, t}^{(\alpha)}$ and preserves the completely integrable systems. Furthermore, $\Psi_{\Gamma, 0}^{(\alpha)}$ coincides with $\Psi_{\Gamma, 1}^{(\alpha+1)}$ on $X_{\Gamma, 0}^{(\alpha)}=X_{\Gamma, 1}^{(\alpha+1)}$.

Proof. We prove the proposition by induction on $\alpha$. The case $\alpha=1$ is proved in Lemma 6.3. Assume that the statement of the proposition holds for $\Psi_{\Gamma, t}^{(\alpha-1)}$. The same argument as in the proof of Lemma 6.3 shows that there exists an open subset $W_{\Gamma, t}^{(\alpha)} \subset X_{\Gamma, t}^{(\alpha)}$ on which the gradient-Hamiltonian flow is defined and that $\phi_{t-t^{\prime}}^{(\alpha)}: W_{\Gamma, t}^{(\alpha)} \rightarrow W_{\Gamma, t^{\prime}}^{(\alpha)}$ is a symplectomorphism preserving $\Psi_{\Gamma, t}^{(\alpha)}$. From the hypothesis of induction, $\Phi_{\Gamma, 1}^{(\alpha)}$ is a completely integrable system, and hence $\Phi_{\Gamma, t}^{(\alpha)}=\left(\phi_{1-t}^{(\alpha)}\right)^{-1^{*}} \Phi_{\Gamma, 1}^{(\alpha)}$ is also a completely integrable system on $W_{\Gamma, t}^{(\alpha)}$. What we need to show is that $W_{\Gamma, t}^{(\alpha)}$ is dense in $X_{\Gamma, t}^{(\alpha)}$.

Let $P=P_{1}^{(\alpha-1)} \cup \cdots \cup P_{\alpha}^{(\alpha-1)}$ be the subdivision given by $d_{1}, \ldots, d_{\alpha-1}$, and suppose that $d_{\alpha}$ is a diagonal of $P_{1}^{(\alpha-1)}$. Then we have

$$
X_{\Gamma, t}^{(\alpha)}=\widetilde{X}_{\Gamma_{1}^{(\alpha-1)}, t}^{(1)} \times \widetilde{\mathrm{Gr}}_{P_{2}^{(\alpha-1)}} \times \cdots \times \widetilde{\mathrm{Gr}}_{P_{\alpha}^{(\alpha-1)}} / / \mathbb{C}_{0}^{*} \times \mathbb{C}_{d_{1}}^{*} \times \cdots \times \mathbb{C}_{d_{\alpha-1}}^{*}
$$

The functions $\widetilde{\lambda}_{\beta, j}(\beta \geq \alpha)$ and $\widetilde{\psi}_{e_{i}}(i=1, \ldots, n-1)$ in $\Psi_{\Gamma, t}^{(\alpha)}$ are induced from $\Psi_{\Gamma_{1}^{(\alpha-1)}, t}^{(1)}$ on $X_{\Gamma_{1}^{(\alpha-1)}, t}^{(1)}$ and $\Psi_{\Gamma_{m}^{(\alpha-1)}}$ on $\operatorname{Gr}_{P_{m}^{(\alpha-1)}}(m \geq 2)$. From Lemma 6.3 and the fact that the Hamiltonian torus action of $\Psi_{\Gamma, t}^{(\alpha)}$ and $\Psi_{\Gamma_{m}^{(\alpha-1)}}$ is defined on an open dense subset of $\operatorname{Gr}_{P_{m}^{(\alpha-1)}}$, the Hamiltonian actions of $\widetilde{\lambda}_{\beta, j}$ and $\widetilde{\psi}_{e_{i}}$ are also defined on an open subset of $X_{\Gamma, t}^{(\alpha)}$. On the other hand, the Hamiltonian action of $\widetilde{\nu}_{\beta}$ is the diagonal $S_{d_{\beta}}^{1}$-action, which is defined everywhere on $X_{\Gamma, t}^{(\alpha)}$ and transverse to the Hamiltonian actions of $\widetilde{\lambda}_{\beta, j}$ and $\widetilde{\mu}_{S_{i}^{1}}$. In particular, $\operatorname{Int}\left(X_{\Gamma, t}^{(\alpha)}\right)$, on which the Hamiltonian $T^{2 n-4}$-action is free, is dense in $X_{\Gamma, t}^{(\alpha)}$, and hence so is $W_{\Gamma, t}^{(\alpha)} \subset X_{\Gamma, t}^{(\alpha)}$.

Since $\Psi_{\Gamma, 0}^{(\alpha)}$ is induced from the integrable systems on $X_{\Gamma_{1}^{(\alpha-1)}, 0}^{(1)}$ and $\mathrm{Gr}_{P_{m}^{(\alpha-1)}}$, the last statement of the proposition follows from Lemma 6.3.

Since the toric degeneration of $\Psi_{\Gamma}: \operatorname{Gr}(2, n) \rightarrow \mathbb{R}^{2 n-4}$ is invariant under the action of maximal torus $T_{U(n)}=\prod_{i=1}^{n} S_{e_{i}}^{1}$, we have the following. 
Corollary 6.5. The toric degeneration $\left(\mathfrak{X}_{\Gamma}^{(\alpha)}, \widetilde{\Psi}_{\Gamma}^{(\alpha)}, \phi^{(\alpha)}\right)$ of $\Psi_{\Gamma}$ induces a toric degeneration of the bending system on $\mathcal{M}_{r}$ associated to $\Gamma$. In particular, $\Delta_{\Gamma}(\boldsymbol{r})=\Phi_{\Gamma}\left(\mathcal{M}_{\boldsymbol{r}}\right)$ is a moment polytope of the central fiber $X_{\Gamma, 0} / / T_{U(n)}$.

EXAMPLE 6.6. Let $n=5$, and assume that the side lengths $r_{1}, \ldots, r_{5}$ are close to each other as in Example 3.3. Then $\mathcal{M}_{\boldsymbol{r}}$ is isomorphic to $\mathbb{C P}^{2}$ blown up at four points in general position. It follows from Figure 2 that the central fiber $X_{\Gamma, 0} / / T_{U(n)}$ is $\mathbb{C P}^{2}$ blown up four times at two pairs of infinitely near points.

\section{$\S 7$. Properties of $\Gamma$}

Let $X_{\Gamma}:=X_{\Gamma, 0}$ be the toric variety obtained as the central fiber of the toric degeneration of $\operatorname{Gr}(2, n)$ associated with a triangulation $\Gamma$ of the reference polygon $P$.

Lemma 7.1. The torus fixed-point set in the toric variety $X_{\Gamma} \subset \mathbb{P}\left(\bigwedge^{2} \mathbb{C}^{n}\right)$ consists of points $p_{k l}=\left[Z_{i j}\right]_{i, j} \in \mathbb{P}\left(\bigwedge^{2} \mathbb{C}^{n}\right)(\{k, l\} \subset\{1, \ldots, n\})$ defined by $Z_{i j}=0$ for all $\{i, j\} \neq\{k, l\}$. In particular, the number of fixed points is $n(n-1) / 2$.

Proof. First note that any torus fixed point in a toric variety is obtained as an intersection of toric divisors. It follows from the description of $X_{\Gamma}$ given in Corollary 5.7 that a toric divisor in $X_{\Gamma}$ is written as

$$
D_{a b}^{P_{m}^{(n-3)}}=\left\{Z_{a b}^{P_{m}^{(n-3)}}=0\right\}
$$

where $a, b \in\left\{e_{1}, \ldots, e_{n}, d_{1}, \ldots, d_{n-3}\right\}$ are a pair of edges in a triangle $P_{m}^{(n-3)}$ in the triangulation

$$
P=P_{1}^{(n-3)} \cup \cdots \cup P_{n-2}^{(n-3)}
$$

of the reference polygon $P$. The image of $D_{a b}^{P_{m}^{(n-3)}}$ in $\mathbb{P}\left(\bigwedge^{2} \mathbb{C}^{n}\right)$ is given by $\bigcap_{i, j}\left\{Z_{i j}=0\right\}$, where the intersection is taken over all $i, j$ such that the path $\gamma(i, j)$ intersects with $a$ and $b$. Then a torus-invariant subvariety $\bigcap D_{a b}^{P_{m}^{(n-3)}}$ is 0 -dimensional exactly when there is a unique pair $(k, l)$ such that $\gamma(k, l)$ does not intersect $a, b \in\left\{e_{1}, \ldots, e_{n}, d_{1}, \ldots, d_{n-3}\right\}$.

Suppose that $|\boldsymbol{r}|=n$ so that the Kähler form on $\operatorname{Gr}(2, n)$ represents the first Chern class of $\operatorname{Gr}(2, n)$. Then for each fixed point $p_{k l}$ in $X_{\Gamma}$, we have

$$
\widetilde{\psi}_{e_{i}}\left(p_{k l}\right)= \begin{cases}n & \text { if } i=k \text { or } i=l \\ 0 & \text { otherwise }\end{cases}
$$


and

$$
\widetilde{\nu}_{\alpha}\left(p_{k l}\right)= \begin{cases}0 & \text { if the path } \gamma(k, l) \text { intersects } d_{\alpha}, \\ n & \text { otherwise. }\end{cases}
$$

This shows that the vertices of the moment polytope $\Delta_{\Gamma}$ of $X_{\Gamma}$ with respect to this symplectic form are lattice points, so that $\Delta_{\Gamma}$ is an integral polytope.

Definition 7.2. A reflexive polytope $\Delta$ is an integral polytope such that

- $\Delta$ is given by $\Delta=\left\{u \in \mathbb{R}^{N} \mid\left\langle v_{i}, u\right\rangle \geq-1, i=1, \ldots, m\right\}$ for some $v_{1}, \ldots$, $v_{m} \in \mathbb{Z}^{N}$, where $m$ is the number of facets of $\Delta$, and

- $\Delta$ has the unique lattice point 0 in its interior.

Proposition $7.3([\mathrm{~B}])$. The moment polytope of a polarized toric variety is reflexive up to translation if and only if it is a canonically polarized Gorenstein toric Fano variety.

Proposition 7.4. If $|\boldsymbol{r}|=n$, then $\Delta_{\Gamma}$ is a reflexive polytope up to translation by an integral vector. Hence, $X_{\Gamma}$ is a Gorenstein toric Fano variety.

Proof. Set $u_{e_{i}}^{\prime}=u_{e_{i}}-2$ for each side $e_{i}$, and set $u_{d_{\alpha}}^{\prime}=u_{d_{\alpha}}+1-\left|I_{\alpha}\right|$ for a diagonal $d_{\alpha}=\sum_{i \in I_{\alpha}} e_{i}$. Then $\left(u_{a}^{\prime}\right)_{a}=0$ is equivalent to $u(a)=1$ for all $a$, where $u(a)$ are the coordinates defined by (4.2) corresponding to lengths of sides and diagonals. Recall that $\Delta_{\Gamma}$ is defined by triangle inequalities $|u(a)-u(b)| \leq u(c) \leq u(a)+u(c)$ for each triangle in the triangulation $\Gamma$. Then $\left(u_{a}^{\prime}\right)_{a}=0$ defines an interior point in $\Delta_{\Gamma}$. We have to show that

- the triangle inequalities have the form $\left\langle v, u^{\prime}\right\rangle \geq-1$ for some integral vector $v \in \mathbb{Z}^{2 n-4}$, and

- $\left(u_{a}^{\prime}\right)_{a}=0$ is the unique interior lattice point in $\Delta_{\Gamma}$.

We divide the proof into steps, as follows.

Step 1. The triangle inequality associated with a triangle consisting of two edges $e_{i}, e_{i+1}$ and a diagonal $d_{\alpha}$ has the form $\left\langle v, u^{\prime}\right\rangle \geq-1$ for some integral vector $v \in \mathbb{Z}^{2 n-4}$.

In this case, one has $I_{\alpha}=\{i, i+1\}$ or $I_{\alpha}=\{1, \ldots, n\} \backslash\{i, i+1\}$, depending on the orientation of $d_{\alpha}$. The triangle inequalities in the first case are given by

$$
\frac{1}{2}\left|u_{e_{i}}^{\prime}-u_{e_{i+1}}^{\prime}\right| \leq \frac{1}{2}\left(u_{e_{i}}^{\prime}+u_{e_{i+1}}^{\prime}\right)-u_{\alpha, 2}^{\prime}+1 \leq \frac{1}{2}\left(u_{e_{i}}^{\prime}+u_{e_{i+1}}^{\prime}\right)+2,
$$

and these are equivalent to

$$
u_{d_{\alpha}}^{\prime} \geq-1, \quad u_{e_{i}}^{\prime}-u_{d_{\alpha}}^{\prime} \geq-1, \quad u_{e_{i+1}}^{\prime}-u_{d_{\alpha}}^{\prime} \geq-1
$$


Similarly, the triangle inequalities for the second case are

$$
u_{e_{i}}^{\prime}+u_{e_{i+1}}^{\prime}+u_{d_{\alpha}}^{\prime} \geq-1, \quad-u_{e_{i}}^{\prime}-u_{d_{\alpha}}^{\prime} \geq-1, \quad-u_{e_{i+1}}^{\prime}-u_{d_{\alpha}}^{\prime} \geq-1 .
$$

Step 2. The triangle inequality associated with a triangle consisting of two diagonals $d_{\alpha}, d_{\beta}$ and a side $e_{j}$ has the form $\left\langle v, u^{\prime}\right\rangle \geq-1$ for some integral vector $v \in \mathbb{Z}^{2 n-4}$.

We may assume that $I_{\beta}=I_{\alpha} \cup\{j\}$, or that $I_{\alpha} \cup I_{\beta}=\{1, \ldots, n\} \backslash\{j\}$ and $I_{\alpha} \cap I_{\beta}=\emptyset$, depending on the choice of orientations of the diagonals. In the first case, we have $d_{\beta}=d_{\alpha}+e_{j}$, and hence the triangle inequalities are

$$
\left|u_{d_{\alpha}}^{\prime}-u_{d_{\beta}}^{\prime}+\frac{1}{2} u_{e_{j}}^{\prime}\right| \leq \frac{1}{2} u_{e_{j}}^{\prime}+1 \leq \sum_{i \in I_{\alpha}} u_{e_{i}}^{\prime}+\frac{1}{2} u_{e_{j}}^{\prime}-u_{d_{\alpha}}^{\prime}-u_{d_{\beta}}^{\prime}+2
$$

which are equivalent to

$$
\begin{aligned}
u_{d_{\beta}}^{\prime}-u_{d_{\alpha}}^{\prime} & \geq-1, \quad u_{e_{j}}^{\prime}+u_{d_{\alpha}}^{\prime}-u_{d_{\beta}}^{\prime} \geq-1, \\
\sum_{i \in I_{\alpha}} u_{e_{i}}^{\prime}-u_{d_{\alpha}}^{\prime}-u_{d_{\beta}}^{\prime} \geq-1 . &
\end{aligned}
$$

The triangle inequalities for the second case are

$$
\begin{array}{r}
-u_{e_{j}}^{\prime}-u_{d_{\alpha}}^{\prime}-u_{d_{\beta}}^{\prime} \geq-1, \\
-u_{d_{\alpha}}^{\prime}+u_{d_{\beta}}^{\prime}+u_{e_{j}}^{\prime}+\sum_{i \in I_{\alpha}} u_{e_{i}}^{\prime} \geq-1 \\
u_{d_{\alpha}}^{\prime}-u_{d_{\beta}}^{\prime}-\sum_{i \in I_{\alpha}} u_{e_{i}}^{\prime} \geq-1 .
\end{array}
$$

Step 3. The triangle inequality associated with a triangle consisting of three diagonals $d_{\alpha}, d_{\beta}, d_{\gamma}=d_{\alpha}+d_{\beta}$ has the form $\left\langle v, u^{\prime}\right\rangle \geq-1$ for some integral vector $v \in \mathbb{Z}^{2 n-4}$.

In this case, we have

$$
\begin{array}{r}
\sum_{i \in I_{\alpha}} u_{e_{i}}^{\prime}-u_{d_{\alpha}}^{\prime}+u_{d_{\beta}}^{\prime}+u_{d_{\gamma}}^{\prime} \geq-1 \\
\sum_{i \in I_{\beta}} u_{e_{i}}^{\prime}+u_{d_{\alpha}}^{\prime}+u_{d_{\beta}}^{\prime}-u_{d_{\gamma}}^{\prime} \geq-1 \\
-u_{d_{\alpha}}^{\prime}-u_{d_{\beta}}^{\prime}+u_{d_{\gamma}}^{\prime} \geq-1 .
\end{array}
$$


If the orientations of $d_{\alpha}, d_{\beta}, d_{\gamma}$ are chosen in such a way that $d_{\alpha}+d_{\beta}+d_{\gamma}=$ 0 , then $I_{\alpha} \cup I_{\beta} \cup I_{\gamma}=\{1, \ldots, n\}$, and hence the triangle inequalities are

$$
\begin{gathered}
u_{d_{\alpha}}^{\prime}-u_{d_{\beta}}^{\prime}-u_{d_{\gamma}}^{\prime}-\sum_{i \in I_{\alpha}} u_{e_{i}}^{\prime} \geq-1, \\
-u_{d_{\alpha}}^{\prime}+u_{d_{\beta}}^{\prime}-u_{d_{\gamma}}^{\prime}-\sum_{i \in I_{\beta}} u_{e_{i}}^{\prime} \geq-1, \\
-u_{d_{\alpha}}^{\prime}-u_{d_{\beta}}^{\prime}+u_{d_{\gamma}}^{\prime}-\sum_{i \in I_{\gamma}} u_{e_{i}}^{\prime} \geq-1,
\end{gathered}
$$

as desired.

Step 4. We have that $\left(u_{a}^{\prime}\right)_{a}=0$ is the unique interior lattice point in $\Delta_{\Gamma}$.

Let $\left(u_{a}^{\prime}\right)$ be an interior lattice point in $\Delta_{\Gamma}$. For a triangle consisting of two edges $e_{i}, e_{i+1}$ and a diagonal $d_{\alpha},(7.1)$ or (7.2) implies that $u_{e_{i}}^{\prime}, u_{e_{i+1}}^{\prime}$, and $u_{d_{\alpha}}^{\prime}$ satisfy

$$
u_{d_{\alpha}}^{\prime} \geq 0, \quad u_{e_{i}}^{\prime}-u_{d_{\alpha}}^{\prime} \geq 0, \quad u_{e_{i+1}}^{\prime}-u_{d_{\alpha}}^{\prime} \geq 0,
$$

or

$$
u_{e_{i}}^{\prime}+u_{e_{i+1}}^{\prime}+u_{d_{\alpha}}^{\prime} \geq 0, \quad-u_{e_{i}}^{\prime}-u_{d_{\alpha}}^{\prime} \geq 0, \quad-u_{e_{i+1}}^{\prime}-u_{d_{\alpha}}^{\prime} \geq 0 .
$$

Then we have $u_{e_{i}}^{\prime}, u_{e_{i+1}}^{\prime} \geq 0$ in either case. Similarly, for a triangle consisting two diagonals $d_{\alpha}, d_{\beta}$ and one side $e_{j}$, we obtain $u_{e_{j}}^{\prime} \geq 0$ from (7.3) or (7.4). Hence, $u_{e_{i}}^{\prime} \geq 0$ for all sides $e_{1}, \ldots, e_{n}$. Combining this with $\sum_{i=1}^{n} u_{e_{i}}^{\prime}=0$, we have $u_{e_{i}}^{\prime}=0$ or, equivalently, $u\left(e_{i}\right)=1$ for all $e_{1}, \ldots, e_{n}$. Note that the coordinate change $\left(u_{d_{\alpha}}^{\prime}\right)_{\alpha} \mapsto\left(u\left(d_{\alpha}\right)\right)_{\alpha}$ restricted to $u_{e_{1}}^{\prime}=\cdots=u_{e_{n}}^{\prime}=0$ is defined over $\mathbb{Z}$. In particular, $\left(u_{d_{\alpha}}^{\prime}\right) \in \mathbb{Z}^{n-3}$ if and only if $\left(u\left(d_{\alpha}\right)\right)_{\alpha} \in \mathbb{Z}^{n-3}$. We take a triangle $P_{1}$ consisting of two sides $e_{i}, e_{i+1}$ and a diagonal $d_{\alpha}$. The triangle inequalities $0=\left|u\left(e_{i}\right)-u\left(e_{i+1}\right)\right|<u\left(d_{\alpha}\right)<u\left(e_{i}\right)+u\left(e_{i+1}\right)=2$ imply that $u\left(d_{\alpha}\right)=1$ or, equivalently, $u_{d_{\alpha}}^{\prime}=0$. Then the $(n-1)$-gon $P \backslash P_{1}$ also has edges with unit lengths. By repeating this process, we obtain $u_{a}^{\prime}=0$ for all $a$.

Let

$$
Y_{\Gamma}=\widetilde{\mathrm{Gr}}_{P_{1}^{(n-3)}} \times \cdots \times \widetilde{\mathrm{Gr}}_{P_{n-2}^{(n-3)}} / /(1,1, \ldots, 1) \mathbb{C}_{0}^{*} \times \mathbb{C}_{d_{1}}^{*} \times \cdots \times \mathbb{C}_{d_{n-3}}^{*}
$$

be the symplectic reduction of $\widetilde{\mathrm{Gr}}_{P_{1}^{(n-3)}} \times \cdots \times \widetilde{\mathrm{Gr}}_{P_{n-2}^{(n-3)}}$ at level $(1,1, \ldots, 1)$. Since $X_{\Gamma}$ is the symplectic reduction of the same space at level $(1,0, \ldots, 0)$, there is a natural map $\pi: Y_{\Gamma} \rightarrow X_{\Gamma}$. 
Proposition 7.5. We have that $\pi: Y_{\Gamma} \rightarrow X_{\Gamma}$ is a small resolution.

Proof. We first show that $Y_{\Gamma}$ is smooth. Recall that the moment map of the $S_{d_{\alpha}}^{1}$-action on $\widetilde{\mathrm{Gr}}_{P_{1}^{(n-3)}} \times \cdots \times \widetilde{\mathrm{Gr}}_{P_{n-2}^{(n-3)}}$ is given by

$$
\mu_{S_{d_{\alpha}}^{1}}=\frac{1}{2}\left(\left|Z_{a_{1} d_{\alpha}}^{P_{a}}\right|^{2}+\left|Z_{a_{2} d_{\alpha}}^{P_{a}}\right|^{2}-\left|Z_{b_{1} d_{\alpha}}^{P_{b}}\right|^{2}-\left|Z_{b_{2} d_{\alpha}}^{P_{b}}\right|^{2}\right)
$$

Then one can see that the $S_{d_{\alpha}}^{1}$-action on $\mu_{S_{d_{\alpha}}^{1}}^{-1}(1)$ is free, so that the symplectic reduction

$$
Y_{\Gamma} \cong \mu_{S_{0}^{1}}^{-1}(1) \cap \mu_{S_{d_{1}}^{1}}^{-1}(1) \cap \cdots \cap \mu_{S_{d_{n-3}}^{1}}^{-1}(1) / S_{0}^{1} \times S_{d_{1}}^{1} \times \cdots \times S_{d_{n-3}}^{1}
$$

is smooth.

The natural morphism $\pi: Y_{\Gamma} \rightarrow X_{\Gamma}$ sends a point $[y] \in Y_{\Gamma}$ for

$$
y \in \mu_{S_{0}^{1}}^{-1}(1) \cap \mu_{S_{d_{1}}^{1}}^{-1}(1) \cap \cdots \cap \mu_{S_{d_{n-3}}^{1}}^{-1}
$$

to $[x] \in X_{\Gamma}$, where $x$ is a point

$$
x \in \overline{O_{y}} \cap\left(\mu_{S_{0}^{1}}^{-1}(1) \cap \mu_{S_{d_{1}}^{1}}^{-1}(0) \cap \cdots \cap \mu_{S_{d_{n-3}}^{1}}^{-1}(0)\right)
$$

in the intersection of the closure of the $\mathbb{C}_{0}^{*} \times \mathbb{C}_{d_{1}}^{*} \times \cdots \times \mathbb{C}_{d_{n-3}}^{*}$-orbit

$$
O_{y} \subset \widetilde{\mathrm{Gr}}_{P_{1}^{(n-3)}} \times \cdots \times \widetilde{\mathrm{Gr}}_{P_{n-2}^{(n-3)}}
$$

of $y$. One can see that

$$
E_{\Gamma}=\left\{[y] \in Y_{\Gamma} \mid Z_{b_{1} d_{\alpha}}^{P_{b}}=Z_{b_{2} d_{\alpha}}^{P_{b}}=0 \text { for some triangle } P_{b}\right\}
$$

is the exceptional set of $\pi$; the morphism $\pi$ is an isomorphism outside $E_{\Gamma}$ since $O_{y}$ is closed if $y \notin E_{\Gamma}$, and $\pi$ is not an isomorphism at $E_{\Gamma}$ since $\pi(y) \in X_{\Gamma}$ for $y \in E_{\Gamma}$ is singular by Proposition 5.8, whereas $Y_{\Gamma}$ is smooth everywhere.

Since $E \subset Y_{\Gamma}$ is of codimension 2, the exceptional locus of $\pi$ does not contain a divisor, and Proposition 7.5 is proved.

Remark 7.6. Propositions 7.4 and 7.5 are not true for toric degenerations of polygon spaces in general. Indeed, the triangulation $\Gamma_{2}$ in Example 3.3 gives a degeneration of the space $\mathcal{M}_{r}$ of pentagons into the Hirzebruch surface $F_{2}$ of degree 2, which is not Fano. If we further assume that $r_{1}=$ $r_{2}$, then the central fiber is the weighted projective plane $\mathbb{P}(1,1,2)$, whose minimal resolution $F_{2} \rightarrow \mathbb{P}(1,1,2)$ is not small. 


\section{$\S 8$. Potential functions}

For a Lagrangian submanifold $L$ in a symplectic manifold, the cohomology group $H^{*}\left(L ; \Lambda_{0}\right)$ has a structure of a weak $A_{\infty}$-algebra (see $[\mathrm{FO}+1]$ ), where $\Lambda_{0}$ is the Novikov ring

$$
\Lambda_{0}=\left\{\sum_{i=0}^{\infty} a_{i} T^{\lambda_{i}} \mid a_{i} \in \mathbb{Q}, \lambda_{i} \in \mathbb{R}_{\geq 0}, \lim _{i \rightarrow \infty} \lambda_{i}=\infty\right\} .
$$

A solution to the Maurer-Cartan equation

$$
\sum_{k=0}^{\infty} \mathfrak{m}_{k}(b, \ldots, b) \equiv 0 \quad \bmod \operatorname{PD}([L])
$$

is called a weak bounding cochain, where $\operatorname{PD}([L])$ is the Poincare dual of the fundamental class $[L]$. The potential function is a map $\mathfrak{P O}: \mathcal{M}(L) \rightarrow \Lambda_{0}$ from the moduli space $\mathcal{M}(L)$ of weak bounding cochains defined by

$$
\sum_{k=0}^{\infty} \mathfrak{m}_{k}(b, \ldots, b)=\mathfrak{P O}(b) \cdot \operatorname{PD}([L]) .
$$

Cho and $\mathrm{Oh}[\mathrm{CO}]$ and Fukaya, Oh, Ohta, and Ono [FO+2] computed the potential functions for Lagrangian torus orbits in toric manifolds. This is generalized in [NNU1] and [NNU2] to an integrable system on a Fano manifold which has a degeneration into the toric moment map on a toric Fano variety admitting a small resolution. From Propositions 7.4 and 7.5, we can apply this result to the toric degeneration of the integrable system $\Psi_{\Gamma}$ on $\operatorname{Gr}(2, n)$ to obtain the following.

TheOREM 8.1. Fix a triangulation $\Gamma$ of the reference polygon, and let $\ell_{i}(u)=\left\langle v_{i}, u\right\rangle-\tau_{i}$ be the affine functions defining $\Delta_{\Gamma}$ :

$$
\Delta_{\Gamma}=\left\{u \in \mathbb{R}^{2 n-4} \mid \ell_{i}(u) \geq 0, i=1, \ldots, m\right\} .
$$

Then for any $u \in \operatorname{Int} \Delta_{\Gamma}$, one has an inclusion $H^{1}\left(L(u) ; \Lambda_{0}\right) \subset \mathcal{M}(L(u))$ for the Lagrangian torus fiber $L(u)=\Psi_{\Gamma}^{-1}(u)$, and the potential function is given by

$$
\mathfrak{P O}_{\Gamma}(L(u), x)=\sum_{i=1}^{m} e^{\left\langle v_{i}, x\right\rangle} T^{\ell_{i}(u)}
$$

for $x \in H^{1}\left(L(u), \Lambda_{0}\right) \cong \Lambda_{0}^{2 n-4}$. 
By setting $y_{a}=e^{x_{a}} T^{u_{a}}$ and $Q=T^{|\boldsymbol{r}|}$, the potential function can be regarded as a Laurent polynomial in $y_{a}$ and $Q$. Since $\Delta_{\Gamma}$ is given by triangle inequalities

$$
\begin{gathered}
-u(a)+u(b)+u(c) \geq 0, \\
u(a)-u(b)+u(c) \geq 0, \\
u(a)+u(b)-u(c) \geq 0
\end{gathered}
$$

in terms of the length coordinates defined in (4.2), the potential function can be written as

$$
\mathfrak{P O}_{\Gamma}=\sum_{\text {triangles }}\left(\frac{y(b) y(c)}{y(a)}+\frac{y(a) y(c)}{y(b)}+\frac{y(a) y(b)}{y(c)}\right),
$$

where $y(a)$ is a Laurent monomial in $y_{e_{i}}^{1 / 2}, y_{d_{\alpha}}$, and $Q$ is defined by

$$
y(a)= \begin{cases}y_{e_{i}}^{1 / 2}, & a=e_{i}(i=1, \ldots, n-1), \\ Q\left(y_{e_{1}} \ldots y_{e_{n-1}}\right)^{-1 / 2}, & a=e_{n}, \\ y_{d_{\alpha}}^{-1} \prod_{i \in I_{\alpha}} y_{e_{i}}^{1 / 2}, & a=d_{\alpha},\end{cases}
$$

and the sum is taken over all triangles in the triangulation $\Gamma$. This is the potential function given in (1.4), and Theorem 1.6 is proved.

Now we consider the relation between the potential functions $\mathfrak{P O}_{\Gamma_{1}}$ and $\mathfrak{P O}_{\Gamma_{2}}$ corresponding to two different triangulations $\Gamma_{1}$ and $\Gamma_{2}$. It suffices to consider the case where $\Gamma_{1}$ is transformed into $\Gamma_{2}$ by a single Whitehead move replacing a diagonal $d$ with $d^{\prime}$. Recall that the piecewise-linear transformation (1.3) sends $\Delta_{\Gamma_{1}}$ to $\Delta_{\Gamma_{2}}$. We define its geometric lift in the sense of $[\mathrm{BZ}]$ by

$$
y\left(d^{\prime}\right)=y(d) \cdot \frac{y\left(a_{1}\right) y\left(a_{4}\right)+y\left(a_{2}\right) y\left(a_{3}\right)}{y\left(a_{1}\right) y\left(a_{2}\right)+y\left(a_{3}\right) y\left(a_{4}\right)},
$$

which means that the tropicalization of this map gives the piecewise-linear transformation (1.3). From (1.3) or a direct computation, (8.1) is also written as

$$
y\left(d^{\prime}\right)=y(d) \cdot \frac{\frac{y\left(a_{1}\right)}{y\left(a_{2}\right)}+\frac{y\left(a_{2}\right)}{y\left(a_{1}\right)}+\frac{y\left(a_{3}\right)}{y\left(a_{4}\right)}+\frac{y\left(a_{4}\right)}{y\left(a_{3}\right)}}{\frac{y\left(a_{1}\right)}{y\left(a_{4}\right)}+\frac{y\left(a_{4}\right)}{y\left(a_{1}\right)}+\frac{y\left(a_{2}\right)}{y\left(a_{3}\right)}+\frac{y\left(a_{3}\right)}{y\left(a_{2}\right)}} .
$$

Then Theorem 8.1 gives the following. 
COROLlARY 8.2. Under the above situation, the potential functions $\mathfrak{P O}_{\Gamma_{1}}$ and $\mathfrak{P O}_{\Gamma_{2}}$ are related by the rational map (8.1).

Proof. The potential function corresponding to $\Gamma_{1}$ is written as

$$
\begin{aligned}
\mathfrak{P O}_{\Gamma_{1}}= & y(d)\left(\frac{y\left(a_{1}\right)}{y\left(a_{2}\right)}+\frac{y\left(a_{2}\right)}{y\left(a_{1}\right)}+\frac{y\left(a_{3}\right)}{y\left(a_{4}\right)}+\frac{y\left(a_{4}\right)}{y\left(a_{3}\right)}\right) \\
& +\frac{y\left(a_{1}\right) y\left(a_{2}\right)+y\left(a_{3}\right) y\left(a_{4}\right)}{y(d)}+F(y),
\end{aligned}
$$

where $F(y)$ is a Laurent polynomial which does not contain $y_{d}$. Since the triangle inequalities for $\Gamma_{1}$ and $\Gamma_{2}$ are the same except for those containing $d$ and $d^{\prime}$, the potential function for $\Gamma_{2}$ is written as

$$
\begin{aligned}
\mathfrak{P O}_{\Gamma_{2}}= & y\left(d^{\prime}\right)\left(\frac{y\left(a_{1}\right)}{y\left(a_{4}\right)}+\frac{y\left(a_{4}\right)}{y\left(a_{1}\right)}+\frac{y\left(a_{2}\right)}{y\left(a_{3}\right)}+\frac{y\left(a_{3}\right)}{y\left(a_{2}\right)}\right) \\
& +\frac{y\left(a_{1}\right) y\left(a_{4}\right)+y\left(a_{2}\right) y\left(a_{3}\right)}{y\left(d^{\prime}\right)}+F(y) .
\end{aligned}
$$

Hence, the coordinate change (8.1) transforms $\mathfrak{P O}_{\Gamma_{1}}$ into $\mathfrak{P \mathfrak { O } _ { \Gamma _ { 2 } }}$.

Theorem 1.7 is a direct consequence of Corollary 8.2.

REMARK 8.3. In the case of flag manifolds, Rusinko [Ru] proved a similar result for string polytopes.

EXAMPLE 8.4. Consider a triangulation $\Gamma$ of a quadrilateral given by $d=e_{1}+e_{2}$. The triangle inequalities for $\Gamma$ are

$$
\begin{array}{r}
\left\langle(1,0,0,-1),\left(u_{e_{1}}, u_{e_{2}}, u_{e_{3}}, u_{d}\right)\right\rangle \geq 0, \\
\left\langle(0,1,0,-1),\left(u_{e_{1}}, u_{e_{2}}, u_{e_{3}}, u_{d}\right)\right\rangle \geq 0, \\
\left\langle(0,0,0,1),\left(u_{e_{1}}, u_{e_{2}}, u_{e_{3}}, u_{d}\right)\right\rangle \geq 0, \\
\left\langle(0,0,-1,-1),\left(u_{e_{1}}, u_{e_{2}}, u_{e_{3}}, u_{d}\right)\right\rangle+|\boldsymbol{r}| \geq 0, \\
\left\langle(-1,-1,0,1),\left(u_{e_{1}}, u_{e_{2}}, u_{e_{3}}, u_{d}\right)\right\rangle+|\boldsymbol{r}| \geq 0, \\
\left\langle(1,1,1,-1),\left(u_{e_{1}}, u_{e_{2}}, u_{e_{3}}, u_{d}\right)\right\rangle-|\boldsymbol{r}| \geq 0 .
\end{array}
$$

Thus, the potential function is

$$
\mathfrak{P O}_{\Gamma}=\frac{y_{e_{1}}}{y_{d}}+\frac{y_{e_{2}}}{y_{d}}+y_{d}+\frac{Q}{y_{e_{3}} y_{d}}+\frac{Q y_{d}}{y_{e_{1}} y_{e_{2}}}+\frac{y_{e_{1}} y_{e_{2}} y_{e_{3}}}{Q y_{d}} .
$$


After the coordinate change

$$
y_{1}^{(1)}=y_{e_{1}}, \quad y_{1}^{(2)}=\frac{y_{e_{1}} y_{e_{2}}}{y_{d}}, \quad y_{2}^{(2)}=y_{d}, \quad y_{2}^{(3)}=\frac{y_{e_{1}} y_{e_{2}} y_{e_{3}}}{Q},
$$

which is a geometric lift of (4.4), the potential function becomes

$$
\mathfrak{P O}_{\Gamma}=y_{2}^{(2)}+\frac{y_{1}^{(1)}}{y_{2}^{(2)}}+\frac{y_{2}^{(3)}}{y_{2}^{(2)}}+\frac{y_{1}^{(2)}}{y_{1}^{(1)}}+\frac{y_{1}^{(2)}}{y_{2}^{(3)}}+\frac{Q}{y_{1}^{(2)}} .
$$

REMARK 8.5. The potential function in (8.3) coincides with the superpotential in [EHX, (B.2)]. More generally, for every $n$, the potential function corresponding to the caterpillar coincides with [EHX, (B.25)] after a coordinate change corresponding to (4.4).

Acknowledgments. We thank Takeo Nishinou for collaboration at an early stage of this research; this paper was originally conceived as a joint project with him. We are also grateful to Hiroshi Konno for valuable discussion, which is reflected in the proof of Theorem 1.2. And we thank one of the referees for carefully reading the manuscript and for suggesting several improvements.

\section{REFERENCES}

[A1] D. Auroux, Mirror symmetry and T-duality in the complement of an anticanonical divisor, J. Gökova Geom. Topol. GGT 1 (2007), 51-91. MR 2386535.

[A2] - "Special Lagrangian fibrations, wall-crossing, and mirror symmetry" in Geometry, Analysis, and Algebraic Geometry: Forty Years of the Journal of Differential Geometry, Surv. Differ. Geom. 13, International Press, Somerville, Mass., 2009, 1-47. MR 2537081.

[B] V. V. Batyrev, Dual polyhedra and mirror symmetry for Calabi-Yau hypersurfaces in toric varieties, J. Algebraic Geom. 3 (1994), 493-535. MR 1269718.

[BZ] A. Berenstein and A. Zelevinsky, Tensor product multiplicities, canonical bases and totally positive varieties, Invent. Math. 143 (2001), 77-128. MR 1802793. DOI 10.1007/s002220000102.

[CO] C.-H. Cho and Y.-G. Oh, Floer cohomology and disc instantons of Lagrangian torus fibers in Fano toric manifolds, Asian J. Math. 10 (2006), 773-814. MR 2282365. DOI 10.4310/AJM.2006.v10.n4.a10.

[EHX] T. Eguchi, K. Hori, and C.-S. Xiong, Gravitational quantum cohomology, Internat. J. Modern Phys. A 12 (1997), 1743-1782. MR 1439892. DOI 10.1142/ S0217751X97001146.

[Fo] P. Foth, Moduli spaces of polygons and punctured Riemann spheres, Canad. Math. Bull. 43 (2000), 162-173. MR 1754021. DOI 10.4153/CMB-2000-024-1.

$[\mathrm{FO}+1] \quad$ K. Fukaya, Y.-G. Oh, H. Ohta, and K. Ono, Lagrangian Intersection Floer Theory: Anomaly and Obstruction, AMS/IP Stud. Adv. Math. 46, Amer. Math. Soc., Providence, 2009. MR 2553465. 
$[\mathrm{FO}+2]-$ Lagrangian Floer theory on compact toric manifolds, I, Duke Math. J. 151 (2010), 23-174. MR 2573826. DOI 10.1215/00127094-2009-062.

$[\mathrm{FO}+3] \longrightarrow$, Lagrangian Floer theory and mirror symmetry on compact toric manifolds, preprint, arXiv:1009.1648v2 [math.SG].

$[\mathrm{FO}+4] \longrightarrow$ Toric degeneration and non-displaceable Lagrangian tori in $S^{2} \times S^{2}$, preprint, arXiv:1002.1660v1 [math.SG].

[GM] I. M. Gel'fand and R. D. MacPherson, Geometry in Grassmannians and a generalization of the dilogarithm, Adv. Math. 44 (1982), 279-312. MR 0658730. DOI 10.1016/0001-8708(82)90040-8.

[GS] V. Guillemin and S. Sternberg, The Gel'fand-Cetlin system and quantization of the complex flag manifolds, J. Funct. Anal. 52 (1983), 106-128. MR 0705993. DOI 10.1016/0022-1236(83)90092-7.

[HK] J.-C. Hausmann and A. Knutson, Polygon spaces and Grassmannians, Enseign. Math. (2) 43 (1997), 173-198. MR 1460127.

[HMM] B. Howard, C. Manon, and J. J. Millson, The toric geometry of triangulated polygons in Euclidean space, Canad. J. Math. 63 (2011), 878-937. MR 2849001. DOI 10.4153/CJM-2011-021-0.

[J] L. C. Jeffrey, Extended moduli spaces of flat connections on Riemann surfaces, Math. Ann. 298 (1994), 667-692. MR 1268599. DOI 10.1007/BF01459756.

[KY] Y. Kamiyama and T. Yoshida, Symplectic toric space associated to triangle inequalities, Geom. Dedicata 93 (2002), 25-36. MR 1934683. DOI 10.1023/A: 1020393910472.

[KM] M. Kapovich and J. J. Millson, The symplectic geometry of polygons in Euclidean space, J. Differential Geom. 44 (1996), 479-513. MR 1431002.

[Kl] A. A. Klyachko, "Spatial polygons and stable configurations of points in the projective line" in Algebraic Geometry and Its Applications (Yaroslavl', 1992), Aspects Math. E25, Vieweg, Braunschweig, 1994, 67-84. MR 1282021.

[Ko] B. Kostant, "Orbits, symplectic structures and representation theory" in Proceedings of the U.S.-Japan Seminar in Differential Geometry (Kyoto, 1965), Nippon Hyoronsha, Tokyo, 1966, 71. MR 0213476.

[MP] J. J. Millson and J. A. Poritz, Around polygons in $\mathbb{R}^{3}$ and $S^{3}$, Comm. Math. Phys. 218 (2001), 315-331. MR 1828984. DOI 10.1007/PL00005557.

[NNU1] T. Nishinou, Y. Nohara, and K. Ueda, Toric degenerations of GelfandCetlin systems and potential functions, Adv. Math. 224 (2010), 648-706. MR 2609019. DOI 10.1016/j.aim.2009.12.012.

[NNU2] , Potential functions via toric degenerations, Proc. Japan Acad. Ser. A Math. Sci. 88 (2012), 31-33. MR 2879356. DOI 10.3792/pjaa.88.31.

[R1] W.-D. Ruan, "Lagrangian torus fibration of quintic hypersurfaces, I: Fermat quintic case" in Winter School on Mirror Symmetry, Vector Bundles and Lagrangian Submanifolds (Cambridge, Mass., 1999), AMS/IP Stud. Adv. Math. 23, Amer. Math. Soc., Providence, 2001, 297-332. MR 1876075.

[R2] - Lagrangian torus fibration of quintic Calabi-Yau hypersurfaces, II: Technical results on gradient flow construction, J. Symplectic Geom. 1 (2002), 435-521. MR 1959057.

[Ru] J. Rusinko, Equivalence of mirror families constructed from toric degenerations of flag varieties, Transform. Groups 13 (2008), 173-194. MR 2421321. DOI 10.1007/s00031-008-9008-y. 
[SS] D. Speyer and B. Sturmfels, The tropical Grassmannian, Adv. Geom. 4 (2004), 389-411. MR 2071813. DOI 10.1515/advg.2004.023.

[T] T. Treloar, The symplectic geometry of polygons in the 3-sphere, Canad. J. Math. 54 (2002), 30-54. MR 1880958. DOI 10.4153/CJM-2002-002-1.

Yuichi Nohara

Faculty of Education

Kagawa University

Takamatsu

Kagawa, 760-8522

Japan

nohara@ed.kagawa-u.ac.jp

Kazushi Ueda

Department of Mathematics

Graduate School of Science

Osaka University

Toyonaka

Osaka, 560-0043

Japan

kazushi@math.sci.osaka-u.ac.jp 